\title{
Optimal solutions of multiplier DEA models
}

\author{
Victor V. Podinovski $\mathbb{D}^{1} \cdot$ Tatiana Bouzdine-Chameeva ${ }^{2}$
}

Accepted: 6 May 2021 / Published online: 9 June 2021

(c) The Author(s) 2021

\begin{abstract}
Conventional models of data envelopment analysis (DEA) are based on the constant and variable returns-to-scale production technologies. Any optimal input and output weights of the multiplier DEA models based on these technologies are interpreted as being the most favorable for the decision making unit (DMU) under the assessment when the latter is benchmarked against the set of all observed DMUs. In this paper we consider a very large class of DEA models based on arbitrary polyhedral technologies, which includes almost all known convex DEA models. We highlight the fact that the conventional interpretation of the optimal input and output weights in such models is generally incorrect, which raises a question about the meaning of multiplier models. We address this question and prove that the optimal solutions of such models show the DMU under the assessment in the best light in comparison to the entire technology, but not necessarily in comparison to the set of observed DMUs. This result allows a clear and meaningful interpretation of the optimal solutions of multiplier models, including known models with a complex constraint structure whose interpretation has been problematic and left unaddressed in the existing literature.
\end{abstract}

JEL C14 $\cdot \mathrm{C} 61 \cdot \mathrm{C} 67 \cdot \mathrm{D} 24$

Keywords Data envelopment analysis $\cdot$ Multiplier model $\cdot$ Polyhedral technology $\cdot$ Input and output weights $\cdot$ Supporting hyperplane

\section{Introduction}

The two conventional models of data envelopment analysis (DEA) are based on the assumption that the production technology is characterized by either constant or variable returns to scale (CRS and VRS), respectively. The CRS and VRS DEA models of Charnes et al. (1978) and Banker et al. (1984), whose earlier statements were introduced in the production theory literature by Afriat (1972), Shephard (1974) and Färe et al. (1983), are linear programs that can be stated in two mutually dual envelopment and multiplier forms.

The interpretation of the CRS and VRS models is wellestablished and depends on whether they are solved in the

Victor V. Podinovski

v.podinovski@lboro.ac.uk

1 School of Business and Economics, Loughborough University, Loughborough LE11 3TU, UK

2 KEDGE Business School, 680 cours de la Libération, 33405 Talence Cedex, France input or output orientation (Førsund 2018). The optimal value of these programs is interpreted as, respectively, the input or output radial efficiency of the decision making unit (DMU) under the assessment, denoted $\mathrm{DMU}_{o}$. From the envelopment perspective, the input or output radial efficiency of $\mathrm{DMU}_{o}$ represents the utmost factor by which the input or output vector of $\mathrm{DMU}_{o}$ can be improved in the given technology.

The dual multiplier CRS model is stated in terms of the input and output weights. As shown by Charnes et al. (1978), any optimal weights of this model (often also referred to as the input and output shadow prices) maximize the ratio of the total weighted output to the total weighted input of $\mathrm{DMU}_{o}$, under the condition that similar ratios calculated for all observed DMUs do not exceed the value of 1. Following this interpretation, the optimal input and output weights are regarded as the most favorable for $\mathrm{DMU}_{o}$ (represent $\mathrm{DMU}_{o}$ in the best light) when it is benchmarked against the set of all observed DMUs.

A similar interpretation is used for the optimal weights of the multiplier VRS model. This model also includes an additional dual variable corresponding to the normalizing equality (convexity constraint) in the envelopment model. 
Banker and Thrall (1992) show that the range of optimal values of this variable defines the type of returns to scale exhibited by the projection of $\mathrm{DMU}_{o}$ on the boundary of the VRS technology. The dual characterization of the CRS and VRS production technologies and optimal solutions of the multiplier models based on them have been extensively studied in the literature - see, e.g., Briec and Leleu (2003), Färe and Primont (1995), Olesen and Petersen $(1996,2015)$ and Zelenyuk (2013).

In recent years, a number of new DEA models have been developed based on various assumptions about the production technology. Most of these technologies are modeled as polyhedral sets and are referred to as polyhedral technologies (Podinovski et al. 2016). The class of polyhedral technologies including the standard CRS and VRS technologies of Charnes et al. (1978) and Banker et al. (1984), the non-increasing and non-decreasing returns-to-scale technologies (Färe et al. 1983, Seiford and Thrall 1990), and their extensions by production trade-offs which are dual terms to weight restrictions (Podinovski 2004b). Further examples include the hybrid returns-to-scale technology based on the idea of selective proportionality between certain subsets of inputs and outputs (Podinovski 2004a), technologies with multiple component processes, including those with joint or shared inputs and outputs (Cherchye et al. 2013, Podinovski et al. 2018), and models based on (selective) weak disposability of inputs and outputs (Mehdiloo and Podinovski 2019). Finally, the underlying technologies of various network DEA models (Färe and Grosskopf 2000) are also polyhedral.

Similar to the conventional CRS and VRS models, DEA models employing any polyhedral technology can be stated in two mutually dual envelopment and multiplier forms. While the interpretation of the envelopment models is usually straightforward and similar to the case of the CRS and VRS models, the interpretation of the multiplier models and their optimal solutions (vectors of shadow prices) is often problematic. Of course, duality theory and standard interpretation of shadow prices apply to all such models and their optimal solutions. However, typically, such solutions include not only the vectors of input and output weights but also a number of other variables dual to the often complex constraints of the envelopment model. What the constraints of the multiplier models mean, whether the optimal input and output weights (and the optimal values of any other dual variables) are the most favorable for the $\mathrm{DMU}_{o}$ and, if so, then exactly in what sense, is often unclear.

One example of polyhedral technology that illustrates the described difficulty is the standard CRS or VRS technology expanded by the specification of production trade-offs (dual to weight restrictions in the multiplier models). Such tradeoffs represent permissible substitutions between certain input and output quantities, and their specification expands the production technology (Podinovski 2004b). Based on the earlier example of Podinovski and Athanassopoulos (1998), in a further development, Podinovski (2016) shows that the optimal input and output weights of such models may not necessarily be the most favorable for $\mathrm{DMU}_{o}$ among all nonnegative weights when it is benchmarked against the observed DMUs. Therefore, the conventional interpretation of the optimal weights does not apply to models based on such polyhedral technologies. ${ }^{1}$

In this paper we give a further example based on the nonincreasing returns-to-scale technology in which the traditional interpretation of optimal solutions of the multiplier model is clearly incorrect. These examples show that we do not have a general meaningful explanation of the multiplier models for arbitrary polyhedral technologies, except for the CRS and VRS models and a few other models for which alternative bespoke interpretations, that apply only to a particular model, have been developed in the literature (see, e.g., Cherchye et al. 2013, Podinovski et al. 2018). ${ }^{2}$

A new way to interpret optimal solutions of the multiplier model was recently suggested in a special case of polyhedral technology, namely, for the VRS and CRS technologies expanded by production trade-offs. Podinovski (2016) proved that the optimal input and output weights of the corresponding multiplier models are the most favorable for $\mathrm{DMU}_{o}$ among all nonnegative weights, in comparison not to the set of observed DMUs (as already shown by Podinovski and Athanassopoulos (1998)) but in comparison to the infinite set of all DMUs in the technology expanded by the trade-offs.

In this paper we generalize this result to the whole class of polyhedral technologies and obtain a unifying interpretation of the multiplier models based on them. We also reinterpret optimal solutions of multiplier models in terms of profit maximization, and show that the optimal input and output weights of the multiplier model based on any polyhedral technology maximize the profit efficiency of $\mathrm{DMU}_{o}$ in comparison to all DMUs in the technology.

To achieve this, we first develop a transformation of the multiplier model based on any polyhedral technology to a different linear model whose structure is transparent and

\footnotetext{
${ }^{1}$ Podinovski (2016) also considers a different interpretation of the most favorable weights that, in addition to being nonnegative, are also required to satisfy the weight restrictions dual to the production tradeoffs. This interpretation, stated as Interpretation 1 on page 921 of Podinovski (2016), is correct only for weight restrictions of certain types. It is specific to models with weight restrictions and is not considered in this paper.

${ }^{2}$ For example, Podinovski et al. (2018) consider a technology with multiple component processes characterized by shared inputs and outputs. They show that an optimal solution of the corresponding multiplier model can be seen as the most favorable for $\mathrm{DMU}_{o}$ in the sense that it identifies the most favorable input and output weights and also the proportions in which the shared inputs and outputs are allocated to the component processes.
} 
amenable to interpretation. We further suggest two additional transformations to the linear fractional and maximin models in which the efficiency of $\mathrm{DMU}_{o}$ is measured by the ratio of its weighted output and input combinations. Using these restated models, we prove that, irrespective of the often complex structure of the original multiplier model, its optimal solutions are the most favorable for $\mathrm{DMU}_{o}$ both in the conventional sense explored by Charnes et al. (1978) and Banker et al. (1984), and in the sense of profit maximization, but with the caveat that $\mathrm{DMU}_{o}$ is benchmarked against the entire technology and not against the set of observed DMUs. For example, for any polyhedral cone technology, the optimal input and output weights (which are generally only a part of the longer vector representing an optimal solution) maximize the ratio of the total weighted output to the total weighted input of $\mathrm{DMU}_{o}$ and, alternatively, the profit of $\mathrm{DMU}_{o}$, in comparison to all DMUs in the technology, but not necessarily in comparison to the set of observed DMUs as would be the case for the conventional CRS model.

The new interpretation of optimal solutions obtained in this paper applies to any polyhedral technology and does not contradict the established interpretations for the CRS and VRS models. The optimal weights of the CRS and VRS multiplier models are the most favorable for the $\mathrm{DMU}_{o}$ under the assessment if it is benchmarked against the finite set of observed DMUs (as in the standard interpretation) and also against the entire CRS or VRS technology (as in the new interpretation obtained in this paper).

We proceed as follows. In Section 2, we consider a motivational example based on the HRS technology of Podinovski (2004a). We show that the optimal solutions of the standard multiplier model based on this technology do not allow a straightforward interpretation. In the subsequent sections, we obtain their interpretation, and the interpretation in the case of any other polyhedral technology, by restating the standard linear multiplier models in three new forms.

We obtain this new universal interpretation not for an individual technology, but for the whole class of polyhedral technologies, examples of which were highlighted above, in one unifying development. To enable this new development, in Section 3, we first introduce a general statement of polyhedral technology and the envelopment and multiplier models based on it. In Section 4, we develop a universal transformation that restates the multiplier model based on an arbitrary polyhedral technology in a transparent linear form. In section 5, we discuss the hierarchy of three forms of the multiplier model in the standard case of VRS technology, which clarifies the direction of further theoretical investigation. In Section 6, we obtain transformations of the multiplier model to the linear fractional and maximin forms. All these transformed models allow us to prove that any optimal input and output weights (which are often only a part a full optimal solution of the original multiplier model) are the most favorable for $\mathrm{DMU}_{o}$ when the latter is benchmarked against all DMUs in the entire technology. In Section 7, we prove that optimal solutions of the multiplier CRS and VRS models of Charnes et al. (1978) and Banker et al. (1984) can be interpreted in two ways, as being the most favorable for $\mathrm{DMU}_{o}$ if it is benchmarked against the set of observed DMUs and also if it is benchmarked against the entire technology generated by such DMUs. In Section 8 , we consider a numerical example that illustrates the theoretical results of this paper. In Section 9, we present a concluding summary of the main results. All mathematical proofs are given in Appendix A. In Appendix B, we show the usefulness of our results for the interpretation of multiplier models based on three different polyhedral technologies.

\section{Motivational example}

The hybrid returns-to-scale (HRS) technology was introduced and axiomatically defined by Podinovski (2004a) as a generalization of the standard CRS technology of Charnes et al. (1978) based on the concept of selective proportionality. The HRS technology models a situation in which only a subset of outputs and a subset of inputs are assumed to be mutually proportional (scalable), while the other inputs and outputs are not a part of this proportion.

Let $\left(X_{j}, Y_{j}\right) \in \mathbb{R}_{+}^{m+s}$ be the observed DMUs, where $j=$ $1, \ldots, n$. The input and output vectors $X_{j}$ and $Y_{j}, j=1, \ldots, n$, are the columns of the input and output matrices $\bar{X}$ and $\bar{Y}$ whose dimensions are $m \times n$ and $s \times n$, respectively.

We now consider the rows of matrices $\bar{X}$ and $\bar{Y}$ corresponding to the nonproportional inputs and outputs, and replace them by zero rows. Denote the resulting $m \times n$ and $s \times n$ matrices $\tilde{X}$ and $\tilde{Y}$, respectively. The columns $\tilde{X}_{j}$ and $\tilde{Y}_{j}, j=1, \ldots, n$, of these matrices are the corresponding input and output vectors $X_{j}$ and $Y_{j}$ whose nonproportional components are replaced by zeros.

As proved by Podinovski (2004a), technology $\mathcal{T}_{\text {HRS }}$ is the set of all DMUs $(X, Y) \in \mathbb{R}_{+}^{m+s}$ for which there exist vectors $\lambda, \mu, \nu \in \mathbb{R}^{n}$ and slack vectors $S_{X} \in \mathbb{R}^{m}, S_{Y} \in \mathbb{R}^{s}$ and $S_{\lambda} \in \mathbb{R}^{n}$, such that

$$
\begin{aligned}
& \bar{X} \lambda+\tilde{X} \mu-\tilde{X} \nu+S_{X}=X, \\
& \bar{Y} \lambda+\tilde{Y} \mu-\bar{Y} \nu-S_{Y}=Y, \\
& \lambda-\nu-S_{\lambda}=0, \\
& 1^{\top} \lambda=1, \\
& \lambda, \mu, \nu, S_{X}, S_{Y}, S_{\lambda} \geq 0 .
\end{aligned}
$$


In conditions (1), the components of vector $\mu$ allow the selected inputs and outputs of the observed DMUs represented by the columns of matrices $\tilde{X}$ and $\tilde{Y}$ to be scaled up. Similarly, the components of vector $\nu$ allow such inputs and outputs to be scaled down. (As shown by Podinovski (2004a), in the latter scenario we need to replace the matrix $\tilde{Y}$ by $\bar{Y}$ which appears in the term $\bar{Y} \nu$ on the left-hand side of conditions (1b).) Conditions (1c) allow contraction of the selected inputs and outputs (assumed proportional) as long as the difference $\lambda_{j}-\nu_{j}$ remains nonnegative for all $j=1, \ldots, n$, which in turn guarantees that the resulting inputs and outputs of the selectively contracted DMUs remain nonnegative.

Consider assessing the input radial efficiency of DMU $\left(X_{o}, Y_{o}\right)$ in the HRS technology. Specifying the inputoriented envelopment model is straightforward. This requires replacing DMU $(X, Y)$ in conditions (1a) and (1b) by $\operatorname{DMU}\left(\theta X_{o}, Y_{o}\right)$, where $\theta$ is a sign-free variable, and minimizing $\theta$ subject to the resulting constraints.

Let $v \in \mathbb{R}^{m}, u \in \mathbb{R}^{s}, \sigma \in \mathbb{R}^{n}$ and $\rho \in \mathbb{R}$ be dual to the constraints of the envelopment HRS model. The input-oriented multiplier HRS models can then be stated as follows:

$$
\begin{aligned}
& \theta^{*}=\max Y_{o}^{\top} u+\rho \\
& \text { subject to } X_{o}^{\top} v=1, \\
& \bar{Y}^{\top} u-\bar{X}^{\top} v+\sigma+1 \rho \leq 0, \\
& \tilde{Y}^{\top} u-\tilde{X}^{\top} v \leq 0, \\
& -\bar{Y}^{\top} u+\tilde{X}^{\top} v-\sigma \leq 0, \\
& u, v, \sigma \geq 0, \rho \text { sign free. }
\end{aligned}
$$

The constraints of program (2) have a complex structure, and direct interpretation of its optimal solutions $(u, v, \sigma, \rho)$ is a challenging task. It may be argued that we should obtain the interpretation of this program by restating it in the linear fractional form, similar to the interpretation of the CRS model by Charnes et al. (1978):

$$
\begin{aligned}
& \theta^{*}=\max \frac{Y_{o}^{\top} u+\rho}{X_{o}^{\top} v} \\
& \frac{Y_{j}^{\top} u+\sigma_{j}+\rho}{X_{j}^{\top} v} \leq 1, \quad \forall j=1, \ldots, n, \\
& \frac{\tilde{Y^{\top}} u}{\tilde{X}_{j}^{\top} v} \leq 1, \quad \forall j=1, \ldots, n, \\
& \frac{Y_{j}^{\top} u+\sigma_{j}}{X^{\top}{ }_{j} v} \geq 1, \quad \forall j=1, \ldots, n, \\
& u, v, \sigma \geq 0, \rho \text { sign free. }
\end{aligned}
$$

It is clear that the restatement of program (2) in the linear fractional form (3) has not made the interpretation any easier. $^{3}$

Note that it would be erroneous to simply state that the optimal solutions to the multiplier models (2) and (3) represent DMU $\left(X_{o}, Y_{o}\right)$ in the best light compared to the set of observed DMUs, for several reasons. First, the constraints of these programs are stated not only for the observed DMUs $\left(X_{j}, Y_{j}\right)$ but also for the DMU-like structures $\left(\tilde{X}_{j}, \tilde{Y}_{j}\right)$ and $\left(\tilde{X}_{j}, Y_{j}\right)$ as seen in the conditions of program (3). These DMU-like structures are not observed DMUs but can be seen as parts of them. Second, the optimal solutions include vector $\sigma$ and scalar $\rho$ whose role is unclear.

Third, it is known that the input and output weights that are the most favorable for DMU $\left(X_{o}, Y_{o}\right)$ in relation to the observed DMUs are those obtained from the standard multiplier CRS or VRS models. (The meaning of this statement is slightly different in the two models. ${ }^{4}$ ) Optimal solutions of the HRS model (2) are not generally optimal in the CRS or VRS models, and for this reason cannot be the most favorable for DMU $\left(X_{o}, Y_{o}\right)$ in the same sense.

In summary, the standard HRS multiplier model (2) does not allow a clear interpretation of its optimal solutions, and the question arises: what is the meaning of such optimal solutions?

The described difficulties with the HRS technology are not unique and arise in the case of many other technologies, especially those whose statements involve complex conditions. We consider additional examples of such technologies in Appendix B.

In this paper, we address the noted difficulties and explain the meaning of optimal solutions of the multiplier models. We do this in a single development, by considering a general statement of technology as a polyhedral set. We then prove that, regardless of the specific conditions and

\footnotetext{
${ }^{3}$ To see the relationship between programs (2) and (3), let $\left(u^{\prime}, v^{\prime}, \sigma^{\prime}, \rho^{\prime}\right)$ be any feasible (or optimal) solution of the latter program. Then, for any $\alpha>0,\left(\alpha u^{\prime}, \alpha v^{\prime}, \alpha \sigma^{\prime}, \alpha \rho^{\prime}\right)$ is also a feasible (respectively, optimal) solution of (3). (This follows from the observation that the multiplier $\alpha$ appears in the numerators and denominators of all ratio terms of program (3) and cancels out.) All such solutions form an open ray from the origin through $\left(u^{\prime}, v^{\prime}, \sigma^{\prime}, \rho^{\prime}\right)$. The value of the objective function of program (3) on this ray is constant. Program (2) replaces the feasible region of program (3) by a single representative from each such ray which satisfies the normalizing equality $X_{o}^{\top} v=1$. For all such representatives, the linear fractional objective function of program (3) becomes the linear objective function of program (2).

${ }^{4}$ In comparison to the multiplier CRS model, the VRS model incorporates an additional variable dual to the normalizing (convexity) constraint of the envelopment program. As discussed in Sections 4 and 7, this leads to two related but slightly different interpretations of the optimal solutions of the CRS or VRS models as the most favorable for the DMU $\left(X_{o}, Y_{o}\right)$.
} 
complexity of their statements, the optimal solutions of the multiplier model based on any polyhedral technology, including the HRS technology, are the most favorable for DMU $\left(X_{o}, Y_{o}\right)$ when it is compared not to the finite set of observed DMUs but to the infinite set of all DMUs in the technology. (We further illustrate the difference between the two interpretations using an example in Section 8.) This new universal interpretation cannot be observed from the standard multiplier model such as (2), and we need to develop alternative multiplier statements that make this new interpretation clear.

\section{DEA models for polyhedral technologies}

\subsection{Polyhedral technologies}

Consider production technology $\mathcal{T} \subset \mathbb{R}_{+}^{m+s}$ with $m$ inputs and $s$ outputs. To be specific, we assume that $\mathcal{T}$ satisfies the assumption of free (strong) disposability of all inputs and outputs (see, e.g., Färe et al. 1985). The case in which not all inputs and outputs are freely disposable is similar and is not discussed.

Most of the convex production technologies developed in the DEA literature are polyhedral sets. Examples of such technologies were highlighted in Section 1. Using the general form of polyhedral technologies suggested by Podinovski et al. (2016), any freely disposable technology $\mathcal{T}$ is stated as the set of all DMUs $(X, Y) \in \mathbb{R}_{+}^{m+s}$ for which there exist a vector $\hat{\lambda} \in \mathbb{R}^{q}$ and vectors of input and output slacks $S_{X} \in \mathbb{R}^{m}$ and $S_{Y} \in \mathbb{R}^{s}$ such that:

$$
\begin{aligned}
& \hat{X} \hat{\lambda}+S_{X}=X, \\
& \hat{Y} \hat{\lambda}-S_{Y}=Y, \\
& \hat{U} \hat{\lambda}=U_{o}, \\
& \hat{\lambda}, S_{X}, S_{Y} \geq 0 .
\end{aligned}
$$

In the above statement, $\hat{X}, \hat{Y}$ and $\hat{U}$ are matrices of dimensions $m \times q, s \times q$, and $p \times q$, respectively, and $U_{o}$ is a constant vector of dimension $p$. The conditions (4c) are optional and are not always specified. Below we refer to them as the parameter conditions. These conditions are homogeneous if $U_{o}$ is a zero vector and nonhomogeneous otherwise.

Conditions (4) identify a general form in which any freely disposable polyhedral technology can be stated. In each special case of a particular technology, the matrices $\hat{X}$, $\hat{Y}$ and $\hat{U}$, vector $U_{o}$ and the dimensions $p$ and $q$ are different. As an illustration, consider the following examples.

Let $\mathcal{T}$ be the conventional VRS technology of Banker et al. (1984). In this case, the columns of matrices $\hat{X}$ and $\hat{Y}$ are the input and output vectors of the observed DMUs, respectively. The parameter condition (4c) is the single equality $1^{\top} \hat{\lambda}=1$, i.e., $\hat{U}$ is a vector row of ones of dimension $n$, and $U_{o}$ is a scalar equal to 1 . In the case of the CRS technology of Charnes et al. (1978), the equality (4c) is not used.

For many other polyhedral technologies, the structure of matrices $\hat{X}, \hat{Y}$ and $\hat{U}$ used in conditions (4) is more complex. Consider, for example, the HRS technology defined by conditions (1). This technology is a polyhedral technology and its statement (1) is a special case of (4).

Indeed, we can view the combined vector $\left(\lambda, \mu, \nu, S_{\lambda}\right)$ in (1) as the vector $\hat{\lambda}$ in (4). We now restate equalities (1a) and (1b) as follows:

$$
\begin{aligned}
& \bar{X} \lambda+\tilde{X} \mu-\tilde{X} \nu+\bar{O}_{X} S_{\lambda}+S_{X}=X, \\
& \bar{Y} \lambda+\tilde{Y} \mu-\bar{Y} \nu+\bar{O}_{Y} S_{\lambda}-S_{Y}=Y,
\end{aligned}
$$

where $\bar{O}_{X}$ and $\bar{O}_{Y}$ are zero $m \times n$ and $s \times n$ matrices, respectively. It is now clear that equality (1a) corresponds to equality (4a) in which $\hat{X}$ is the combined matrix $\left[\bar{X}, \tilde{X},-\tilde{X}, \bar{O}_{X}\right]$ of dimension $m \times q$, where $q=4 n$. Similarly, condition (1b) corresponds to condition (4b) in which $\hat{Y}$ is the combined matrix $\left[\bar{Y}, \tilde{Y},-\bar{Y}, \bar{O}_{Y}\right]$ of dimension $s \times q$. The equalities (1c) and (1d) are parameter conditions (4c) in which the vector $U_{o}$ is of dimension $n+1$. Its first $n$ components are equal to zero, and its last component is equal to $1 .^{5}$

Two further examples of polyhedral technologies with a complex structure are considered in Appendix B.

Remark 1 Throughout the paper, we distinguish between the matrices $\hat{X}$ and $\hat{Y}$ used in the general statement (4) of a polyhedral technology, and the matrices $\bar{X}$ and $\bar{Y}$ formed by the input and output vectors of the $n$ observed DMUs, as in the statement (1) of the HRS technology. We also distinguish between the intensity vector $\lambda$ used to combine observed DMUs (i.e., the columns of matrices $\bar{X}$ and $\bar{Y}$ ) and the generally longer vector $\hat{\lambda}$ used to combine the columns of matrices $\hat{X}$ and $\hat{Y}$ in (4).

As shown by the case of HRS technology, $\bar{X}$ and $\bar{Y}$ are generally only submatrices of $\hat{X}$ and $\hat{Y}$, although they coincide with the latter in the case of standard VRS and CRS technologies. As another example, in models with weight restrictions, the matrices $\hat{X}$ and $\hat{Y}$ incorporate columns representing the input and output vectors of the

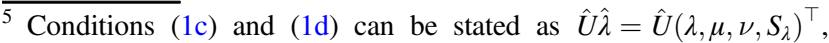
where the matrix $\hat{U}$ is of dimension $(n+1) \times 4 n$ and is defined as follows. Its first $n$ rows correspond to condition (1c) and form the combined $n \times 4 n$ matrix $\left[I_{n}, O_{n},-I_{n},-I_{n}\right]$, where $I_{n}$ and $O_{n}$ are the $n \times$ $n$ identity and zero matrices, respectively. The last row of matrix $\hat{U}$ corresponds to condition (1d). Its first $n$ elements are equal to 1 and the last $3 n$ elements are zeros.
} 
observed DMUs as well as the vectors representing production trade-offs dual to the weight restrictions (Podinovski 2004b).

\subsection{Envelopment and multiplier DEA models}

To be specific, consider assessing the input radial efficiency of $\mathrm{DMU}_{o} \in \mathcal{T}$, whose input and output vectors are $X_{o} \neq 0$ and $Y_{o} \neq 0$, respectively. The case of output radial efficiency is similar and is not discussed.

The input radial efficiency of $\mathrm{DMU}_{o}$ is the optimal value $\theta^{*}$ of the envelopment model

$\theta^{*}=\min \left\{\theta \mid\left(\theta X_{o}, Y_{o}\right) \in \mathcal{T}\right\}$.

Using (4), we restate this envelopment program as follows:

$\theta^{*}=\min \theta$

subject to $\theta X_{o}-\hat{X} \hat{\lambda}-S_{X}=0$,

$\hat{Y} \hat{\lambda}-S_{Y}=Y_{o}$

$\hat{U} \hat{\lambda}=U_{o}$,

$\hat{\lambda}, S_{X}, S_{Y} \geq 0, \theta$ sign free.

Because of the arbitrary nature of technology $\mathcal{T}$, we have to assume specifically that the optimal value of program (5) is finite (and therefore is attained) and that $\theta^{*}>0$. Under this assumption, which should be valid in any meaningful application, all inputs and outputs of the input radial projection $\left(\theta^{*} X_{o}, Y_{o}\right)$ are nonnegative and satisfy conditions (4), together with any optimal solution $\hat{\lambda}^{*}, S_{X}^{*}$ and $S_{Y}^{*}$ of program (5). Therefore, $\left(\theta^{*} X_{o}, Y_{o}\right) \in \mathcal{T}$.

The dual to program (5) is the following multiplier model:

$\theta^{*}=\max Y_{o}^{\top} u+U_{o}^{\top} w$

subject to $X_{o}^{\top} v=1$,

$\hat{Y}^{\top} u-\hat{X}^{\top} v+\hat{U}^{\top} w \leq 0$

$u, v \geq 0, w$ sign free vector.

In model (6), the vectors $v \in \mathbb{R}_{+}^{m}$ and $u \in \mathbb{R}_{+}^{s}$ and the vector of sign-free variables $w \in \mathbb{R}^{p}$ are dual to constraints (5b), (5c) and (5d), respectively. If the parameter condition (5d) is not specified, its dual vector $w$ and the corresponding terms in model (6) are omitted.

It is worth noting that the structure of the multiplier model (6) may be sufficiently complex and make its interpretation problematic. An example is the multiplier HRS model (2) which is a special case of model (6). As highlighted in Section 2, the interpretation of the multiplier HRS model is unclear.

In the next sections, we overcome the problem with the interpretation of model (6) by restating it in several more transparent forms.

\section{Restated linear program}

In this and the next sections we explore the interpretation of optimal solutions of the linear multiplier model (6) by transforming it to the three new useful forms: linear, linear fractional and maximin models. In contrast to the standard multiplier model (6), all three new models have intuitively clear feasible regions which allows straightforward interpretation of their optimal solutions. This series of transformations extends the earlier idea developed by Podinovski et al. (2016) for the special case of CRS and VRS technologies expanded by production trade-offs, to the general case of an arbitrary polyhedral technology.

\subsection{The general case}

Consider the input-oriented multiplier model (6) based on any polyhedral technology $\mathcal{T}$. To facilitate the interpretation of its optimal solutions, we introduce the following program which has an infinite number of linear constraints:

$\varphi_{1}^{*}=\max Y_{o}^{\top} u+u_{0}$

subject to $X_{o}^{\top} v=1$,

$Y^{\top} u-X^{\top} v+u_{0} \leq 0, \forall(X, Y) \in \mathcal{T}$

$u, v \geq 0, u_{0}$ sign free.

To establish a relationship between the standard multiplier program (6) and the above program (7), let $(u, v, w)$ be any feasible solution to the multiplier model (6). Define the scalar

$u_{0}=U_{o}^{\top} w$.

We now generalize the result obtained by Podinovski et al. (2016) for the special case of the VRS technology expanded by production trade-offs to the case of an arbitrary polyhedral technology $\mathcal{T}$ :

Theorem 1 If solution $(u, v, w)$ is feasible in program (6), then solution $\left(u, v, u_{0}\right)$ is feasible in program (7). Furthermore, if $\left(u^{*}, v^{*}, w^{*}\right)$ is optimal in $(6)$, then $\left(u^{*}, v^{*}, u_{0}^{*}\right)$ is 
optimal in (7). The maximum in program (7) is attained, and we have $\theta^{*}=\varphi_{1}^{*}$.

It is worth highlighting the differences between the original multiplier program (6) with a finite number of constraints and its restatement (7) with the infinite number of constraints. The former program is stated in terms of the output and input weights $(u, v)$ and the vector $w$ dual to the parameter conditions $(5 \mathrm{~d})$. Because the constraints of program (6) often have a complex structure, a direct interpretation of this program and its optimal solutions may be problematic.

In contrast, the structure of program (7) is much more transparent. The complex statement (4) of technology $\mathcal{T}$ explicitly incorporated by the standard multiplier model (6) is employed implicitly in program (7) through the intuitively clear (but not really operational) condition $(X, Y) \in$ $\mathcal{T}$ of constraints (7c). Also note that the structure of program (7) is similar to the structure of the standard VRS model of Banker et al. (1984). The only difference is that, in the latter, the full technology $\mathcal{T}$ in constraints $(7 \mathrm{c})$ is replaced by the set of observed DMUs.

Remark 2 It may seem surprising that the matrices $\hat{X}, \hat{Y}$ and $\hat{U}$, and the vector $U_{o}$ that define technology $\mathcal{T}$ by conditions (4) and are used in the standard multiplier model (6) apparently "disappear" in program (7). As already noted, these conditions are still incorporated in program (7), via the condition $(X, Y) \in \mathcal{T}$ in the inequalities $(7 \mathrm{c})$.

To give an intuitive explanation of such transformation, suppose that we sample $a$ very large number $n$ of DMUs $\left(X_{j}, Y_{j}\right)$ from the polyhedral technology $\mathcal{T}$ stated as in (4). We can consider the standard VRS technology generated by this large number of sampled DMUs as a suitable approximation of technology $\mathcal{T}$. Instead of assessing the input radial efficiency of $\mathrm{DMU}_{o}$ in technology $\mathcal{T}$ by solving program (6), we assess it in the approximate technology, by solving the standard input-oriented VRS multiplier program. It is similar to program (7), except for conditions (7c) which are replaced by the standard VRS conditions:

$Y_{j}^{\top} u-X_{j}^{\top} v+u_{0} \leq 0, \forall j=1, \ldots, n$

Because $n$ is very large, we can think of these last conditions as an approximation of condition (7c) stated for every DMU $(X, Y) \in \mathcal{T}$. We now see that, regardless of the complexity of the statement (4) of technology $\mathcal{T}$, program (7) always has the same simple structure similar to the standard VRS model. The statement of technology $\mathcal{T}$ is now incorporated in the conditions $(X, Y) \in \mathcal{T}$ of program (7).

In loose words, we can say that solving the multiplier program (6) based on any polyhedral technology $\mathcal{T}$ simultaneously solves the multiplier model (7) based on the VRS technology generated by the infinite number of all DMUs in technology $\mathcal{T}$.

Of course, the given explanation is not rigorous and does not substitute the precise statement of Theorem 1 and its formal proof. In fact, from the given intuitive explanation it may appear that programs (6) and (7) are equivalent and that their sets of feasible and optimal solutions are the same after the transformation (8) is applied. This is not so, and Theorem 1 only states that any feasible and optimal solution of program (6) is, after the transformation (8), feasible and optimal in program (7), respectively. The fact that the converse is generally not true was demonstrated by Podinovski (2015, Example 2) in the case of CRS technology expanded by production trade-offs.

The optimal value $\theta^{*}$ of both envelopment and multiplier programs (5) and (6) is interpretable as the input radial efficiency of $\mathrm{DMU}_{o}$ in technology $\mathcal{T}$. Theorem 1 suggests an alternative interpretation of any optimal solution $\left(u^{*}, v^{*}, w^{*}\right)$ of program (6) in terms of profit maximization, consistent with the economics literature (see, e.g., Färe and Grosskopf 2004).

Let $u$ and $v$ be regarded as the output and input prices. Then the difference $Y^{\top} u-X^{\top} v$ is the profit generated by $\operatorname{DMU}(X, Y)$. At optimality, at least one constraint in (7c) is satisfied as equality, as otherwise we could increase the optimal output prices $u$ or the scalar $u_{0}$, and the value of the objective function (7a), which contradicts the assumption of optimality. Therefore, the negated sign-free term $-u_{0}$ represents the maximum profit across all DMUs in technology $\mathcal{T}$ (and not only observed DMUs).

Taking into account (7b), we restate the objective function in (7a) as $1-\left[\left(-u_{0}\right)-\left(Y_{o}^{\top} u-X_{o}^{\top} v\right)\right]$. Maximizing this function is equivalent to minimizing the difference between the maximum profit $-u_{0}$ and the actual profit $Y_{o}^{\top} u-X_{o}^{\top} v$ generated by $\mathrm{DMU}_{o}$. By Theorem 1 , the weights $u^{*}$ and $v^{*}$ in any optimal solution $\left(u^{*}, v^{*}, w^{*}\right)$ of program (6) maximize the profit of $\mathrm{DMU}_{o}$ in comparison to the maximum profit of all DMUs in technology $\mathcal{T}$, equal to $-u_{0}^{*}=-U_{o}^{\top} w^{*}$, subject to the cost-normalizing condition $X_{o}^{\top} v=1$.

Furthermore, the difference

$$
1-\theta^{*}=-u_{0}^{*}-\left(Y_{o}^{\top} u^{*}-X_{o}^{\top} v^{*}\right)
$$

is equal to the shortfall of the profit of $\mathrm{DMU}_{o}$ compared to the maximum profit $-u_{0}^{*}$. $\mathrm{DMU}_{o}$ is profit efficient if $1-\theta^{*}$ $=0$, i.e., $\theta^{*}=1$. Therefore, the weights $u^{*}$ and $v^{*}$ are the most favorable for the profit efficiency of $\mathrm{DMU}_{o}$ when it is benchmarked against all DMUs in $\mathcal{T}$. The example given in Section 8 shows that this is different from the conventional interpretation according to which $\mathrm{DMU}_{o}$ is benchmarked against the observed DMUs only, and that the conventional interpretation is generally incorrect in the case of an arbitrary polyhedral technology. 
Remark 3 As a variant of the given interpretation, we can also view the optimal value $\theta^{*}$ of programs (5) and (6) as the exact upper bound representing the attainable profit efficiency of $\mathrm{DMU}_{o}$ if information about the actual input and output prices becomes available. ${ }^{6}$ Indeed, suppose that all DMUs face the same known prices $v^{\prime}$ and $u^{\prime}$ normalized by condition (7b). Denote $-u_{0}^{\prime}=\max _{(X, Y) \in \mathcal{T}}\left\{Y^{\top} u^{\prime}-X^{\top} v^{\prime}\right\}$, the maximum profit across all DMUs in technology $\mathcal{T}$ for such prices. Then the triplet $\left(u^{\prime}, v^{\prime}, u_{0}^{\prime}\right)$ satisfies inequalities (7c) and is a feasible solution of program (7). By Theorem 1 , we have $\theta^{*}=\varphi_{1}^{*}$. Because the latter is the optimal value of program (7), it follows that $\theta^{*} \geq Y_{o}^{\top} u^{\prime}+u_{0}^{\prime}$. Therefore, $\theta^{*}$ is an upper bound on the profit indicator $Y_{o}^{\top} u+u_{0}$ that can in principle be achieved by $\mathrm{DMU}_{o}$, taking into account all possible input and output prices normalized by condition (7b). This upper bound $\theta^{*}$ is exact and is attained at the prices $u^{*}$ and $v^{*}$ taken from any optimal solution $\left(u^{*}, v^{*}, w^{*}\right)$ of program (6).

Furthermore, as follows from (9), the difference $1-\theta^{*}$ is interpretable as the optimal (lowest) shortfall of the profit of $\mathrm{DMU}_{o}$ compared to the maximum profit achieved across all DMUs in technology $\mathcal{T}$, considering all possible (normalized) input and output prices. Therefore, $1-\theta^{*}$ represents the exact lower bound on the actual shortfall of the profit of $\mathrm{DMU}_{o}$ if the information about the actual prices becomes available. For any actual normalized prices, the shortfall of the profit of $\mathrm{DMU}_{o}$ can never be smaller than $1-\theta^{*}$. This lower bound is attained for any optimal prices $u^{*}$ and $v^{*}$ of program (6).

The following result generalizes the known fact established by Banker et al. (1984) for the VRS technology, to an arbitrary polyhedral technology. It shows that any optimal solution of program (6) defines a supporting hyperplane to technology $\mathcal{T}$ at the input radial projection $\left(\theta^{*} X_{o}, Y_{o}\right)$ of the DMU $\left(X_{o}, Y_{o}\right)$. Note the nontrivial way in which the intercept $u_{0}^{*}$ of this hyperplane is calculated.

Proposition 1 Let $\left(u^{*}, v^{*}, w^{*}\right)$ be any optimal solution of program (6). In accordance with (8), define $u_{0}^{*}=U_{o}^{\top} w^{*}$. (Alternatively, and more generally, let $\left(u^{*}, v^{*}, u_{0}^{*}\right)$ be any optimal solution of program (7). ${ }^{7}$ ) Then the set of all pairs $(X, Y) \in \mathbb{R}^{m+s}$ that satisfy the equation

$Y^{\top} u^{*}-X^{\top} v^{*}+u_{0}^{*}=0$

\footnotetext{
6 The authors are grateful to the anonymous reviewer for suggesting this interpretation.

7 According to Theorem 1, every optimal solution $\left(u^{*}, v^{*}, w^{*}\right)$ of program (6) corresponds to an optimal solution $\left(u^{*}, v^{*}, u_{0}^{*}\right)$ of program (7), where $u_{0}^{*}$ is calculated by (8). However, not every optimal solution of program (7) may generally be obtained in this way-see Example 2 given by Podinovski et al. (2016). Proposition 1 is true for any optimal solution of program (7), including any solution obtained from the optimal solution of program (6) by formula (8).
}

is a supporting hyperplane to technology $\mathcal{T}$ at the input radial projection $\left(\theta^{*} X_{o}, Y_{o}\right)$. The vector $\left(u^{*},-v^{*}\right)$ is the normal vector of this supporting hyperplane, and $u_{0}^{*}$ is a constant scalar. For any $\operatorname{DMU}(X, Y) \in \mathcal{T}$, we have $Y^{\top} u^{*}-X^{\top} v^{*}+u_{0}^{*} \leq 0$. For the input radial projection $(X, Y)=\left(\theta^{*} X_{o}, Y_{o}\right) \in \mathcal{T}$, this last inequality is satisfied as equality.

Remark 4 If $\mathcal{T}$ is the VRS technology of Banker et al. (1984), the vector $U_{o}$ in the parameter condition (4c) has a single component equal to 1 and, by formula (8), we have $u_{0}^{*}=w^{*}$. As follows from Banker and Thrall (1992) (see also Førsund and Hjalmarsson 2004), the range of optimal values of $u_{0}$ determines the type of returns to scale (RTS) exhibited by the projection $\left(\theta^{*} X_{o}, Y_{o}\right)$. Podinovski et al. (2016) generalize this result and prove that the range of optimal values of the scalar $u_{0}^{*}=U_{o}^{\top} w^{*}$ defines the type of RTS in an arbitrary polyhedral technology. However, why this relatively unintuitive formula is responsible for the RTS characterization has so far remained unclear. Proposition 1 resolves this issue by demonstrating that $u_{0}^{*}$ is the constant (intercept) of the supporting hyperplane to the technology evaluated at the projected DMU, the role of which in the VRS technology has already been demonstrated by Banker and Thrall (1992).

Let us consider this in greater detail. As follows from the general result of Podinovski et al. (2016, Endnote 14), if $\mathrm{DMU}_{o}$ is both input and output radial efficient ${ }^{8}$ in technology $\mathcal{T}$, the range of optimal values of $u_{0}^{*}=U_{o}^{\top} w^{*}$ defines the left-hand and right-hand scale elasticities $\varepsilon^{-}\left(X_{o}\right.$, $\left.Y_{o}\right)$ and $\varepsilon^{+}\left(X_{o}, Y_{o}\right)$ evaluated at $\mathrm{DMU}_{o}$ and, therefore, its RTS characterization.

More precisely, let $u_{0}^{\min }$ and $u_{0}^{\max }$ be, respectively, the minimum and maximum values of the term $U_{o}^{\top} w$ taken over the set of all optimal solutions to the multiplier model (6). (Identifying $u_{0}^{\min }$ and $u_{0}^{\max }$ requires solving two linear programs.) Then

$\varepsilon^{+}\left(X_{o}, Y_{o}\right)=\frac{1}{1-u_{0}^{\min }}, \quad \varepsilon^{-}\left(X_{o}, Y_{o}\right)=\frac{1}{1-u_{0}^{\max }}$.

The above formulae allow infinite values of $\varepsilon^{+}\left(X_{o}, Y_{o}\right)$ and $\varepsilon^{-}\left(X_{o}, Y_{o}\right)$, obtained when their denominators are equal to zero. Theorem 1 in Podinovski et al. (2016) and its application to the evaluation of scale elasticity discussed in Section 3.3 of the same paper show that, in an arbitrary polyhedral technology $\mathcal{T}$, these cases occur if and only if any marginal proportional increase or, respectively, reduction of the input vector $X_{o}$ of the $\mathrm{DMU}_{o}$ leads outside the technology. For this reason,

\footnotetext{
${ }^{8}$ It obviously suffices that $\mathrm{DMU}_{o}$ be strongly efficient. The output radial efficiency is required for a correct definition of the one-sided scale elasticities evaluated at $\mathrm{DMU}_{o}$. Furthermore, if $\mathrm{DMU}_{o}$ is output radial efficient but input radial inefficient, the one-sided scale elasticities calculated by formulae (11) and the corresponding type of RTS would characterize not the $\mathrm{DMU}_{o}$ but its input projection $\left(\theta^{*} X_{o}, Y_{o}\right)$.
} 
the corresponding one-sided scale elasticity is undefined. If technology $\mathcal{T}$ is freely disposable (which is assumed in the current paper), the right-hand scale elasticity $\varepsilon^{+}\left(X_{o}, Y_{o}\right)$ is always finite and cannot be negative.

This result is similar to the expressions obtained for the standard VRS technology by Banker and Thrall (1992). The difference is that, in the case of VRS, the terms $u_{0}^{\min }$ and $u_{0}^{\max }$ are the extreme values of the actual component $u_{0}$ of the optimal solution to the multiplier VRS model which is dual to the normalizing (convexity) constraint of the envelopment VRS model. For an arbitrary polyhedral technology, $u_{0}^{\min }$ and $u_{0}^{\max }$ are the extreme values of the term $U_{o}^{\top} w$, where $w$ is the vector of dual variables to the parameter conditions $(4 \mathrm{c})$. This term is reduced to the single dual variable $u_{0}$ in the VRS model.

\subsection{Cone technologies}

Assume that the parameter conditions (4c) are either homogeneous or are not specified. In the former case, the vector $U_{o}$ is a zero vector, and conditions (4c) are replaced by the equality

$$
\hat{U} \hat{\lambda}=0 \text {. }
$$

In both cases it is straightforward to verify that technology $\mathcal{T}$ is a polyhedral cone. Examples of polyhedral cone technologies whose statements do not include the parameter condition (4c) are the standard CRS technology of Charnes et al. (1978) and its extension by production trade-offs developed by Podinovski (2004b). An example of polyhedral cone technology with complex homogeneous parameter conditions (4c) is the cone extension of the technology with multiple component processes (Podinovski et al. 2018, p. 287).

Under the stated assumptions, the multiplier model (6) takes on the following form:

$$
\begin{array}{ll}
\theta^{*}=\max & Y_{o}^{\top} u \\
\text { subject to } & X_{o}^{\top} v=1, \\
& \hat{Y}^{\top} u-\hat{X}^{\top} v+\hat{U}^{\top} w \leq 0, \\
& u, v \geq 0, w \text { sign free vector. }
\end{array}
$$

Taking into account (8), we have $u_{0}=0$ and restate program (7) as follows:

$$
\begin{aligned}
& \psi_{1}^{*}=\max Y_{o}^{\top} u \\
& \text { subject to } X_{o}^{\top} v=1, \\
& Y^{\top} u-X^{\top} v \leq 0, \forall(X, Y) \in \mathcal{T}, \\
& u, v \geq 0 .
\end{aligned}
$$

The following results is a straightforward restatement of Theorem 1 for the cone technologies under the consideration:

Theorem 2 If solution $(u, v, w)$ is feasible in program (13), then $(u, v)$ is feasible in program (14). Furthermore, if $\left(u^{*}, v^{*}, w^{*}\right)$ is optimal in $(13)$, then $\left(u^{*}, v^{*}\right)$ is optimal in (14). The maximum in program (14) is attained, and we have $\theta^{*}=\psi_{1}^{*}$.

We are now in a position to provide interpretation of program (13), which is close to the interpretation of the more general program (6). Namely, let $u$ and $v$ be the output and input prices and the difference $Y^{\top} u-X^{\top} v$ be the profit of DMU $(X, Y)$. We first consider program (14). According to constraints (14c), no $\mathrm{DMU}(X, Y) \in \mathcal{T}$ is allowed to have a positive profit. Furthermore, for any optimal prices $u$ and $v$ of this program, at least one inequality in (14c) is satisfied as equality. (Otherwise, we would be able to increase the vector of optimal output prices $u$ and the objective function (14a), which contradicts the assumption of optimality.) This implies that, at optimality, the maximum profit over all DMUs in the entire technology $\mathcal{T}$ is equal to zero, which is consistent with the assumption of CRS.

Using (14b), we can restate the objective function in (14a) as $\left(Y_{o}^{\top} u-X_{o}^{\top} v\right)+1$. Maximizing this function is equivalent to maximizing the profit $Y_{o}^{\top} u-X_{o}^{\top} v$ of $\mathrm{DMU}_{o}$.

Let $\left(u^{*}, v^{*}, w^{*}\right)$ be any optimal solution of program (13). By Theorem 2, the solution $\left(u^{*}, v^{*}\right)$ is optimal in (14). Therefore, the vectors of prices $u^{*}$ and $v^{*}$ maximize the profit of $\mathrm{DMU}_{o}$ subject to the condition that all DMUs in the entire technology $\mathcal{T}$ have a nonpositive profit and that the cost of the input bundle is normalized by the equality $X_{o}^{\top} v=1$.

Note that program (14) requires that the profit of all DMUs in technology $\mathcal{T}$ be nonpositive. For example, the CRS technology of Charnes et al. (1978) expanded by production trade-offs (Podinovski (2004b) is a cone technology. It includes all DMUs in the CRS technology and their modifications obtained by the application of trade-offs in different proportions. It would generally be incorrect to say that the optimal prices $u$ and $v$ in the corresponding multiplier model maximize the profit of $\mathrm{DMU}_{o}$ under the condition that the profit of all observed DMUs is nonpositive. For this interpretation to be correct, we need the profit of all DMUs (including observed DMUs and their modifications by trade-offs) to be nonpositive.

We can also restate Proposition 1 for the technologies considered in this section as follows:

Proposition 2 Let $\left(u^{*}, v^{*}, w^{*}\right)$ be any optimal solution of program (13). (More generally, let $\left(u^{*}, v^{*}\right)$ be any optimal solution of program (14).) Then the set of all pairs $(X, Y) \in$ 
$\mathbb{R}^{m+s}$ that satisfy the equation

$$
Y^{\top} u^{*}-X^{\top} v^{*}=0
$$

is a supporting hyperplane to technology $\mathcal{T}$ at the input radial projection $\left(\theta^{*} X_{o}, Y_{o}\right)$. The vector $\left(u^{*},-v^{*}\right)$ is the normal vector of this supporting hyperplane. For any DMU $(X, Y) \in \mathcal{T}$, we have $Y^{\top} u^{*}-X^{\top} v^{*} \leq 0$. For the input radial projection $(X, Y)=\left(\theta^{*} X_{o}, Y_{o}\right) \in \mathcal{T}$, this last inequality is satisfied as equality.

\section{The hierarchy of multiplier DEA models}

In the previous section, we discussed the interpretation of the linear multiplier program (6) by restating it in the alternative linear form (7), and their variants (13) and (14) for cone technologies. Our next step is to obtain additional interpretation by restating programs (7) and (14) in two further forms, referred to as the linear fractional and maximin models. To add intuition to this development, in this section we provide preliminary discussion that clarifies the relationship between these three forms of the multiplier model, using the standard VRS technology $\mathcal{T}_{\text {VRS }}$ of Banker et al. (1984) as an example. ${ }^{9}$

Let $\left(X_{j}, Y_{j}\right) \in \mathbb{R}_{+}^{m+s}, j=1, \ldots, n$, be the observed DMUs. To evaluate the input radial efficiency of DMU $\left(X_{o}, Y_{o}\right) \in \mathcal{T}_{\text {VRS }}$, we solve the standard linear multiplier VRS program

$$
\begin{array}{ll}
\tilde{\varphi}_{1}=\max & Y_{o}^{\top} u+u_{0} \\
\text { subject to } & X_{o}^{\top} v=1 \\
& Y_{j}^{\top} u-X_{j}^{\top} v+u_{0} \leq 0, \forall j=1, \ldots, n \\
& u, v \geq 0, u_{0} \text { sign free. }
\end{array}
$$

Also consider the linear fractional program (Banker et al. 1984, program (21)):

$$
\begin{array}{ll}
\tilde{\varphi}_{2}=\max & \left(Y_{o}^{\top} u+u_{0}\right) / X_{o}^{\top} v \\
\text { subject to } & \left(Y_{j}^{\top} u+u_{0}\right) / X_{j}^{\top} v \leq 1, \forall j=1, \ldots, n \\
& u, v \geq 0, u_{0} \text { sign free, }
\end{array}
$$

and the maximin program analogous to the maximin model (stated for the case of CRS) of Cooper et al. (1996) and

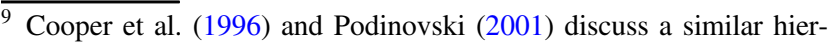
archy of multiplier models in the case of CRS. Its linear, linear fractional and multiplier statements are obtained from programs (15), (16) and (17) by removing the sign-free variable $u_{0}$.
}

Podinovski and Athanassopoulos (1998):

$$
\begin{aligned}
\tilde{\varphi}_{3}= & \max _{\substack{u, v \geq 0, u_{0} \text { sign free }}}\left(\frac{\left(Y_{o}^{\top} u+u_{0}\right) / X_{o}^{\top} v}{\max _{j=1, \ldots, n}\left\{\left(Y_{j}^{\top} u+u_{0}\right) / X_{j}^{\top} v\right\}}\right) \\
= & \max _{u, v \geq 0,}\left(\min _{j=1, \ldots, n}\left\{\frac{\left(Y_{o}^{\top} u+u_{0}\right) / X_{o}^{\top} v}{\left(Y_{j}^{\top} u+u_{0}\right) / X_{j}^{\top} v}\right\}\right) .
\end{aligned}
$$

$u_{0}$ sign free

Let us show that the three programs (15), (16) and (17) are closely related and form a hierarchy in which program (15) is a normalized variant of program (16), which in turn is a normalized variant of program (17).

Program (17) maximizes the efficiency ratio $\left(Y_{o}^{\top} u+\right.$ $\left.u_{0}\right) / X_{o}^{\top} v$ of $\mathrm{DMU}_{o}$ in relation to the maximum of similar ratios of all observed DMUs, under the single condition $u$, $v \geq 0$. Any optimal solution $\left(u^{*}, v^{*}, u_{0}^{*}\right)$ of this program is the most favorable for $\mathrm{DMU}_{o}$ (in the sense of maximizing its efficiency ratio) among all solutions $\left(u, v, u_{0}\right)$ with nonnegative vectors $u$ and $v$ and sign-free scalar $u_{0}$, when $\mathrm{DMU}_{o}$ is benchmarked against the set of observed DMUs.

We now note that, if $\left(u^{\prime}, v^{\prime}, u_{0}^{\prime}\right)$ is a feasible or optimal solution of program (17), then, for any $\alpha>0$ and $\beta>0$, the solution $\left(\alpha u^{\prime}, \beta v^{\prime}, \alpha u_{0}^{\prime}\right)$ is also feasible or, respectively, optimal in (17), because the multipliers $\alpha$ and $\beta$ simultaneously appear in the numerator and denominator of the objective function of this program and cancel out. The value of the objective function for this parametric family of solutions remains the same.

By varying $\alpha$ and $\beta$, we can make the efficiency ratios $\left(Y_{j}^{\top} \alpha u^{\prime}+\alpha u_{0}^{\prime}\right) /\left(X_{j}^{\top} \beta v^{\prime}\right)$ of all DMUs as small (close to zero) or as large as we want. (For example, these ratios become very large as $\beta$ tends to zero.) Because the objective function of program (17) is the same for all solutions $\left(\alpha u^{\prime}, \beta v^{\prime}, \alpha u_{0}^{\prime}\right)$, we can limit its maximization to the subset of the feasible region obtained by the incorporation of the normalizing condition stating that the efficiency ratio $\left(Y_{j}^{\top} u+u_{0}\right) / X_{j}^{\top} v$ of all observed DMUs does not exceed 1 (in which case the maximum of all such ratios is equal to 1). Under this normalizing condition, the denominator in the first line of program (17) becomes equal to 1 , and program (17) is restated as (16). The optimal values of the linear fractional and maximin programs (16) and (17) are equal, and any optimal solution of the former program is optimal in the latter. The converse is true only for the optimal solutions of (17) (members of the parametric family of solutions $\left(\alpha u^{\prime}, \beta v^{\prime}, \alpha u_{0}^{\prime}\right)$ discussed above) for which the maximum of all efficiency ratios $\left(Y_{j}^{\top} u+u_{0}\right) / X_{j}^{\top} v$ across all observed DMUs is equal to 1 .

Now consider any feasible or optimal solution $\left(u^{\prime}, v^{\prime}, u_{0}^{\prime}\right)$ of program (16). For any $\alpha>0$, the solution $\left(\alpha u^{\prime}, \alpha v^{\prime}, \alpha u_{0}^{\prime}\right)$ is also feasible and, respectively, optimal in (16). 
The parametric family of such solutions is an open ray from the origin through the solution $\left(u^{\prime}, v^{\prime}, u_{0}^{\prime}\right)$. The objective function of program (16) remains constant along this ray. We next note that, on each such ray of feasible or optimal solutions, there exists one representative which satisfies the normalizing equality $X_{o}^{\top} v=1$. Therefore, we can limit the maximization of the objective function of program (16) over its entire feasible region by its maximization over the set of such representatives only. This results in program (15). The linear fractional objective function of program (16) becomes a linear objective function of program (15).

The optimal values of the linear and linear fractional programs (15) and (16) are equal, and any optimal solution of the former program is optimal in the latter. The converse is true only for the optimal solutions of program (16) that satisfy the normalizing condition $X_{o}^{\top} v=1$. Such solution obviously exists in each parametric family of optimal solutions $\left(\alpha u^{\prime}, \alpha v^{\prime}, \alpha u_{0}^{\prime}\right)$ of program (16).

In summary, we have shown that programs (15) and (16) can be viewed as progressively normalized variants of the maximin program (17). Any optimal solution of program (15) is also optimal in program (16), and any optimal solution of the latter is also optimal in program (17). The fact that the maximin program (17) is not normalized and maximizes the efficiency ratio of $\mathrm{DMU}_{o}$ against all observed DMUs subject to the single constraint $u, v \geq 0$ makes the interpretation of its optimal solutions, as well as optimal solutions of programs (15) and (16) that are also optimal in (17), straightforward.

In the next section, we show that the discussed hierarchy of multiplier models can be adapted to the modified multiplier model (7) with the infinite number of constraints. It turns out that the latter model also allows the linear fractional and maximin statements useful for interpretation of its optimal solutions.

\section{Further interpretations}

Below, we continue the transformation of the multiplier model (6) started in Section 4, to two further alternative statements, and consider the interpretation of optimal solutions arising from them. The relation between these models is similar to the hierarchy of the three multiplier VRS models discussed in Section 5.

\subsection{The linear fractional model: the general case}

Similar to the standard VRS model discussed in Section 5, we can restate program (7) in the linear fractional form. The resulting program has an infinite number of constraints:

$\varphi_{2}^{*}=\max \left(Y_{o}^{\top} u+u_{0}\right) / X_{o}^{\top} v$ subject to $\left(Y^{\top} u+u_{0}\right) / X^{\top} v \leq 1, \forall(X, Y) \in \mathcal{T}$,

$u, v \geq 0, u_{0}$ sign free.

Theorem 3 If solution $\left(u^{*}, v^{*}, w^{*}\right)$ is optimal in program (6), then $\left(u^{*}, v^{*}, u_{0}^{*}\right)$ is optimal in program (18), where $u_{0}^{*}$ is found by formula (8). The maximum in program (18) is attained, and we have $\theta^{*}=\varphi_{2}^{*}$.

The following statement is a useful analog of Proposition 1.

Proposition 3 For the projected DMU $\left(\theta^{*} X_{o}, Y_{o}\right) \in \mathcal{T}$ and for any optimal solution $\left(u^{*}, v^{*}, u_{0}^{*}\right)$ of program (18), the inequality (18b) is satisfied as equality, i.e.,

$\left(Y_{o}^{\top} u^{*}+u_{0}^{*}\right) /\left(\theta^{*} X_{o}^{\top} v^{*}\right)=1$.

By Theorem 3, any optimal solution $\left(u^{*}, v^{*}, w^{*}\right)$ to the multiplier model (6) maximizes the efficiency ratio $\left(Y_{o}^{\top} u+\right.$ $\left.u_{0}\right) / X_{o}^{\top} v$ of $\mathrm{DMU}_{o}$, where the scalar $u_{0}$ is calculated by formula (8), with respect to the efficiency ratios of all DMUs in technology $\mathcal{T}$, under the condition that no such ratio exceeds the value of 1 . Furthermore, according to Proposition 3, the latter condition can be replaced by the condition that the maximum of all efficiency ratios across all DMUs in the technology is equal to 1 . This maximum is attained at the input radial projection $\left(\theta^{*} X_{o}, Y_{o}\right) \in \mathcal{T}$.

The above interpretation is different from the traditional interpretation of optimal solutions in the conventional multiplier VRS model stated in the linear fractional form (16). In the latter model, the inequalities (18b) are required only for the finite number of observed DMUs, and not for all DMUs in the technology.

To see the economic meaning of Theorem 3, let $u$ and $v$ be interpreted as the output and input prices. Because at optimality at least one of the constraints (18b) is satisfied as equality, the negated sign-free term $-u_{0}$ is interpretable as the maximum profit across all DMUs in the technology. Restate the objective function in (18a) as follows:

$1-\frac{\left(-u_{0}\right)-\left(Y_{o}^{\top} u-X_{o}^{\top} v\right)}{X_{o}^{\top} v}$.

The second term in (19) is the Nerlovian profit indicator of $\mathrm{DMU}_{o}$ normalized by the input combination $X_{o}^{\top} v$ (Chambers et al. 1998, Färe and Grosskopf 2004). According to Theorem 3, the optimal prices $u^{*}$ and $v^{*}$ in any optimal solution $\left(u^{*}, v^{*}, w^{*}\right)$ of program (6) minimize this profit indicator, i.e., maximize the profit efficiency of $\mathrm{DMU}_{o}$ in comparison to all DMUs in technology $\mathcal{T}$. 
For these optimal prices, the maximum profit across all DMUs in $\mathcal{T}$ is equal to $-u_{0}^{*}=-U_{o}^{\top} w^{*}$.

\subsection{The maximin model: the general case}

The efficiency ratios of all DMUs in program (18) are bounded above by 1 . It may be argued that this condition complicates the interpretation of its optimal solutions. Below we remove this condition by restating program (18) in the maximin form:

$$
\begin{aligned}
\varphi_{3}^{*} & =\max _{\substack{u, v \geq 0, u_{0} \text { sign free }}}\left(\frac{\left(Y_{o}^{\top} u+u_{0}\right) / X_{o}^{\top} v}{\sup _{(X, Y) \in \mathcal{T}}\left\{\left(Y^{\top} u+u_{0}\right) / X^{\top} v\right\}}\right) \\
& =\max _{\substack{u, v \geq 0, u_{0} \text { sign free }}}\left(\inf _{(X, Y) \in \mathcal{T}}\left\{\frac{\left(Y_{o}^{\top} u+u_{0}\right) / X_{o}^{\top} v}{\left(Y^{\top} u+u_{0}\right) / X^{\top} v}\right\}\right) .
\end{aligned}
$$

Note that, in contrast to the linear fractional model (18), program (20) does not specify any upper bounds on the efficiency ratios of the DMUs.

Theorem 4 If solution $\left(u^{*}, v^{*}, w^{*}\right)$ is optimal in program (6), then $\left(u^{*}, v^{*}, u_{0}^{*}\right)$ is optimal in program (20), where $u_{0}^{*}$ is found by formula (8). The maximum in program (20) is attained, and we have $\theta^{*}=\varphi_{3}^{*}$.

Theorem 4 allows us to give an interpretation of any optimal solution of program (6) in terms that are independent of the normalizing conditions of the linear and linear fractional programs (7) and (18). Namely, any optimal solution $\left(u^{*}, v^{*}, w^{*}\right)$ to the multiplier model (6) maximizes the efficiency ratio of $\mathrm{DMU}_{o}$ in comparison to the maximum of the efficiency ratios of all DMUs in technology $\mathcal{T}$, under the single condition $u, v \geq 0$, without the upper bounds on such ratios specified in the linear fractional model (18) and without the additional input-normalizing condition $X_{o}^{\top} v=1$ of the linear model (7).

\subsection{Interpretations for cone technologies}

Consider a special case in which $\mathcal{T}$ is a cone technology for which the parameter condition (4c) is either not specified or is the homogeneous condition (12). This allows us to restate the results obtained for the general technology in a simpler form.

Taking into account formula (8) in which $U_{o}$ is a zero vector, we restate the general linear fractional program (18) as follows:

$\psi_{2}^{*}=\max Y_{o}^{\top} u / X_{o}^{\top} v$

subject to $Y^{\top} u / X^{\top} v \leq 1, \forall(X, Y) \in \mathcal{T}$,

$u, v \geq 0$.
Theorem 5 If solution $\left(u^{*}, v^{*}, w^{*}\right)$ is optimal in program (13), then $\left(u^{*}, v^{*}\right)$ is optimal in program (21). The maximum in program (21) is attained, and we have $\theta^{*}=\psi_{2}^{*}$.

The following statement is a useful analog of Proposition 2.

Proposition 4 For the projected DMU $\left(\theta^{*} X_{o}, Y_{o}\right) \in \mathcal{T}$ and for any optimal weights $\left(u^{*}, v^{*}\right)$ of program (21), the inequality (21b) is satisfied as equality, i.e.,

$\left(Y_{o}^{\top} u^{*}\right) /\left(\theta^{*} X_{o}^{\top} v^{*}\right)=1$.

Program (14) can also be restated as the maximin program which does not specify an upper bound on the efficiency ratios:

$$
\begin{aligned}
\psi_{3}^{*} & =\max _{u, v \geq 0}\left(\frac{Y_{o}^{\top} u / X_{o}^{\top} v}{\sup _{(X, Y) \in \mathcal{T}}\left\{Y^{\top} u / X^{\top} v\right\}}\right) \\
& =\max _{u, v \geq 0}\left(\inf _{(X, Y) \in \mathcal{T}}\left\{\frac{Y_{o}^{\top} u / X_{o}^{\top} v}{Y^{\top} u / X^{\top} v}\right\}\right) .
\end{aligned}
$$

Theorem 6 If solution $\left(u^{*}, v^{*}, w^{*}\right)$ is optimal in program (13), then $\left(u^{*}, v^{*}\right)$ is optimal in program (22). The maximum in program (22) is attained, and we have $\theta^{*}=\psi_{3}^{*}$.

Let $\left(u^{*}, v^{*}, w^{*}\right)$ be any optimal solution of program (13). As follows from Theorems 5 and 6 , the optimal weights $\left(u^{*}, v^{*}\right)$ in this solution maximize the efficiency ratio $Y_{o}^{\top} u / X_{o}^{\top} v$ of $\mathrm{DMU}_{o}$ compared to the maximum of such ratios of all DMUs in technology $\mathcal{T}{ }^{10}$ This statement is valid if the efficiency ratios are bounded above by 1 as in the linear fractional program (21), or if no such bounds are specified as in the maximin program (22). In this sense, the optimal weights $\left(u^{*}, v^{*}\right)$ are the most favorable for $\mathrm{DMU}_{o}$ when it is benchmarked against all DMUs in the technology. ${ }^{11}$

The above interpretation is different from the conventional interpretation of the linear fractional CRS model (Charnes et al. 1978) according to which the efficiency ratio of $\mathrm{DMU}_{o}$ is explicitly maximized only in comparison to the observed DMUs.

\footnotetext{
${ }^{10}$ In the economics literature, the ratio $Y^{\top} u / X^{\top} v$ is often referred to as the return to the dollar of the firm $(X, Y)$ for the given input and output prices $v$ and $u$ (Färe and Grosskopf 2004). The weights $\left(u^{*}, v^{*}\right)$ taken from any optimal solution $\left(u^{*}, v^{*}, w^{*}\right)$ of program (13) can therefore be interpreted as the most favorable for representing the return to the dollar of $\mathrm{DMU}_{o}$ in comparison to all DMUs in technology $\mathcal{T}$.

${ }^{11}$ Program (21) is also stated in Lemma 1 of Kuosmanen et al. (2004) for the general case in which $\mathcal{T}$ is a (not necessarily polyhedral) cone technology. However, this result does not imply the exact relationship between the optimal solutions of this program and the standard multiplier model (13) in the sense explored by our Theorem 5 , together with Theorems 2 and 6.
} 


\section{The special case of VRS and CRS technologies}

In this section, we formally show that, for the multiplier VRS and CRS models, both the traditional and new interpretations of their optimal solutions are correct.

\subsection{The VRS technology}

Let $\mathcal{T}_{\text {VRS }}$ be the standard VRS technology of Banker et al. (1984). As shown in Section 5, any optimal solution $\left(u^{*}, v^{*}, u_{0}^{*}\right)$ of the standard multiplier VRS program (15) is optimal in the corresponding linear fractional program (16) and is, in turn, optimal in the maximin program (17). This last program maximizes the efficiency ratio $\left(Y_{o}^{\top} u+\right.$ $\left.u_{0}\right) / X_{o}^{\top} v$ of $\mathrm{DMU}_{o}$ when it is benchmarked against the set of observed DMUs. Therefore, any optimal solution $\left(u^{*}, v^{*}, u_{0}^{*}\right)$ of the standard multiplier VRS model (15) is the most favorable for $\mathrm{DMU}_{o}$ when it is compared to the set of observed DMUs only.

It is interesting to note that, although the optimal solution $\left(u^{*}, v^{*}, u_{0}^{*}\right)$ is identified by program (15) under the two normalizing conditions discussed in Section 5 (the efficiency ratio of all DMUs is bounded above by 1 and the input weights are normalized by the equality $X_{o}^{\top} v=1$ ), this solution is also optimal in the maximin program (17) which does not have any such conditions. Therefore, the solution $\left(u^{*}, v^{*}, u_{0}^{*}\right)$ is the most favorable for benchmarking $\mathrm{DMU}_{o}$ against the set of observed DMUs among all triplets $(u, v$, $u_{0}$ ) under the only condition that $u \geq 0$ and $v \geq 0$.

Now consider the statement of Theorem 4. For the VRS technology, the vector $U_{o}$ in the statement (4) is the scalar equal to 1 , and we have $u_{0}^{*}=w^{*}$. According to Theorem 4, the optimal solution $\left(u^{*}, v^{*}, u_{0}^{*}\right)$ of the standard multiplier VRS model (15) is also optimal in program (20) and is therefore the most favorable for $\mathrm{DMU}_{o}$ when it is benchmarked against all DMUs in technology $\mathcal{T}_{\text {VRS }}$. It is worth pointing out that, although the optimal solution $\left(u^{*}, v^{*}, u_{0}^{*}\right)$ satisfies the normalizing conditions of program (15) mentioned above, it is the best solution among all triplets $(u, v$, $u_{0}$ ) such that $u, v \geq 0$, which is the only condition of the maximin program (20).

In summary, we have shown that any optimal solution $\left(u^{*}, v^{*}, u_{0}^{*}\right)$ of the standard multiplier VRS program (15) is simultaneously the most favorable for $\mathrm{DMU}_{o}$ when it is benchmarked against the observed DMUs only and also against all DMUs in the VRS technology generated by these DMUs.

Remark 5 Benchmarking $\mathrm{DMU}_{o}$ against the set of observed DMUs (in the sense of maximizing its efficiency ratio $\left.\left(Y_{o}^{\top} u+u_{0}\right) / X_{o}^{\top} v\right)$ does not depend on the specification of technology $\mathcal{T}$ and requires solving the maximin program
(17) whose statement does not refer to any technology. In contrast, benchmarking $\mathrm{DMU}_{o}$ against the entire technology $\mathcal{T}$ requires solving the maximin program (20) which depends on $\mathcal{T}$. For $\mathcal{T}=\mathcal{T}_{\text {VRS }}$, program (20) is equivalent to (17). Indeed, as shown in Section 5, solving the maximin program (17) is equivalent to solving the linear multiplier VRS model (15) (with a caveat that solving the latter model identifies only the normalized solutions among the multiple optimal solutions of the maximin model). Taking into account that $u_{0}^{*}=w^{*}$, by Theorem 4 , this is in turn equivalent to solving the maximin program (20) with $\mathcal{T}=\mathcal{T}_{\text {VRS }}$.

This observation puts the VRS technology $\mathcal{T}_{\text {VRS }}$ in a unique position in that it allows the two interpretations discussed above. Namely, benchmarking $\mathrm{DMU}_{o}$ against the set of observed DMUs is equivalent to benchmarking $\mathrm{DMU}_{o}$ against the technology $\mathcal{T}_{\mathrm{VRS}}$ generated by such observed DMUs.

\subsection{The CRS technology}

The case of CRS technology is similar. Let $\mathcal{T}_{\text {CRS }}$ be the standard CRS technology of Charnes et al. (1978). As mentioned in Footnote 9, similar to the multiplier VRS model, the linear multiplier CRS model can also be stated in the linear fractional and maximin forms. These three forms are models (15), (16) and (17) from which we remove the sign-free variable $u_{0}$.

Suppose that the solution $\left(u^{*}, v^{*}\right)$ is optimal in the linear CRS model (model (15) with $u_{0}$ removed). Then $\left(u^{*}, v^{*}\right)$ is optimal in the other two forms, including the maximin model obtained from program (17) by removing variable $u_{0}$. The meaning of the last program is clear: the optimal solution $\left(u^{*}\right.$, $v^{*}$ ) maximizes the efficiency ratio $Y_{o}^{\top} u / X_{o}^{\top} v$ of $\mathrm{DMU}_{o}$ when it is benchmarked against the set of observed DMUs. Because the solution $\left(u^{*}, v^{*}\right)$ is optimal in the maximin model, the weights $u$ and $v$ are the best for $\mathrm{DMU}_{o}$ among all nonnegative weights, and not only among the normalized feasible solutions of the linear multiplier program.

Similarly, by Theorem 6 , the solution $\left(u^{*}, v^{*}\right)$ is also optimal in the maximin program (22) where $\mathcal{T}=\mathcal{T}_{\text {CRS }}$. This means that it maximizes the efficiency ratio $Y_{o}^{\top} u / X_{o}^{\top} v$ of $\mathrm{DMU}_{o}$ when this DMU is benchmarked against the entire technology $\mathcal{T}_{\text {CRS }}$.

We have shown that, for any optimal solution $\left(u^{*}, v^{*}\right)$ of the standard multiplier CRS program, both interpretations are correct. Namely, any such solution is the most favorable for $\mathrm{DMU}_{o}$ (in the sense of maximization of the efficiency ratio $Y_{o}^{\top} u / X_{o}^{\top} v$ ) when it is benchmarked against the set of observed DMUs and against the entire CRS technology generated by these DMUs.

We conclude by making a similar observation to Remark 5. Benchmarking $\mathrm{DMU}_{o}$ against the set of observed DMUs 
Table 1 The data set

\begin{tabular}{lll}
\hline DMU & Input & Output \\
\hline$A$ & 2 & 1 \\
$B$ & 3 & 3 \\
$C$ & 4 & 2 \\
$D$ & 1 & 1 \\
\hline
\end{tabular}

(in the sense of maximizing its efficiency ratio $Y_{o}^{\top} u / X_{o}^{\top} v$ ) does not depend on the assumed technology $\mathcal{T}$ and requires solving the appropriately specified maximin program (program (17) with $u_{0}$ removed). In contrast, benchmarking $\mathrm{DMU}_{o}$ in the same sense against the whole technology leads to the maximin program (22) stated with $\mathcal{T}=\mathcal{T}_{\text {CRS }}$. Similar to the case of VRS discussed in Remark 5, it is straightforward to show that these two maximin programs are equivalent. This puts the CRS technology in a unique position in that benchmarking $\mathrm{DMU}_{o}$ against the set of observed DMUs (in the sense of maximizing the efficiency ratio $\left.Y_{o}^{\top} u / X_{o}^{\top} v\right)$ is equivalent to benchmarking $\mathrm{DMU}_{o}$ against the technology $\mathcal{T}_{\text {CRS }}$ generated by such observed DMUs.

\section{Clarifying example}

This example illustrates the difference between the maximization of the efficiency ratio $\left(Y_{o}^{\top} u+u_{0}\right) / X_{o}^{\top} v$ of the $\mathrm{DMU}_{o}$ with respect to the maximum of such ratios taken over all DMUs in the entire technology $\mathcal{T}$ and, alternatively, over the set of observed DMUs only. ${ }^{12}$ It also illustrates a similar difference in the evaluation of profit efficiency of $\mathrm{DMU}_{o}$.

\subsection{Technology}

Consider the non-increasing returns-to-scale (NIRS) technology $\mathcal{T}_{\text {NIRS }}$ with a single input and a single output generated by the three observed DMUs $A, B$ and $C$ shown in Table 1. (The additional DMU $D$ in this table is not considered observed but is used in the discussion below.) For reference, we also consider the standard VRS technology $\mathcal{T}_{\text {VRS }}$ generated by the same three observed DMUs.

To keep consistency with the general statement of polyhedral technology (4), we state technology $\mathcal{T}_{\text {NIRS }}$ as the set of all DMUs $(X, Y) \in \mathbb{R}_{+}^{m+s}$ for which there exist an

\footnotetext{
$\overline{12}$ A similar example is discussed in Podinovski et al. (2016). It involves a cone technology obtained by an extension of the standard CRS technology by production trade-offs. This example demonstrates the difference between the maximum of the efficiency ratio $Y_{o}^{\top} u / X_{o}^{\top} v$ evaluated over the entire technology and over the set of observed DMUs only.
}

intensity vector $\left(\lambda_{A}, \lambda_{B}, \lambda_{C}\right)^{\top}$ and the scalar slacks $S_{X}, S_{Y}$ and $S_{\lambda}$ such that

$$
\begin{aligned}
& 2 \lambda_{A}+3 \lambda_{B}+4 \lambda_{C}+0 S_{\lambda}+S_{X}=X, \\
& 1 \lambda_{A}+3 \lambda_{B}+2 \lambda_{C}+0 S_{\lambda}-S_{Y}=Y, \\
& 1 \lambda_{A}+1 \lambda_{B}+1 \lambda_{C}+1 S_{\lambda}=1,
\end{aligned}
$$

$\lambda_{A}, \lambda_{B}, \lambda_{C}, S_{X}, S_{Y}, S_{\lambda} \geq 0$

It is clear that (23) is a special case of (4). Indeed, vector $\hat{\lambda}$ in (4) corresponds to the extended vector $\hat{\lambda}=$ $\left(\lambda_{A}, \lambda_{B}, \lambda_{C}, S_{\lambda}\right)^{\top}$ in (23). The scalar $U_{o}$ is equal to 1 and the three matrices $\hat{X}, \hat{Y}$ and $\hat{U}$ have a single row and 4 columns, and are defined as follows:

$\hat{X}=(2,3,4,0), \hat{Y}=(1,3,2,0), \hat{U}=(1,1,1,1)$.

Technology $\mathcal{T}_{\text {NIRS }}$ is shown in Fig. 1 as the shaded area below its boundary $O B W$. The smaller VRS technology $\mathcal{T}_{\text {VRS }}$ generated by the same observed DMUs is shown in darker shading.

\subsection{The multiplier models and their solutions}

Consider assessing the input radial efficiency of DMU $C$ in the specified technologies. For technology $\mathcal{T}_{\text {NIRS }}$, the inputoriented multiplier model is stated as follows:

$$
\begin{array}{ll}
\theta^{*}=\max & 2 u+1 u_{0} \\
\text { subject to } & 4 v=1, \\
& 1 u-2 v+1 u_{0} \leq 0, \\
& 3 u-3 v+1 u_{0} \leq 0, \\
& 2 u-4 v+1 u_{0} \leq 0, \\
& v, u \geq 0, u_{0} \leq 0 .
\end{array}
$$

For technology $\mathcal{T}_{\text {VRS }}$, we use the statement (24) from which we remove the condition $u_{0} \leq 0$, i.e., declare $u_{0}$ a sign-free variable.

Program (24) is a special case of the general statement (6) of the multiplier model based on an arbitrary polyhedral technology $\mathcal{T}$. Note that, in line with the statement of program (6), we should be using notation $w$ for the dual variable to the primal equality (23c) and distinguish it from the variable $u_{0}$ defined by the transformation (8) as $u_{0}=U_{o}^{\top} w$. However, because in this example the scalar $U_{o}=1$, we have $w=u_{0}$. To simplify discussion, we use the same notation $u_{0}$ in all models in this example. (Note that the condition $u_{0} \leq 0$ of program (24) is dual to the primal variable $S_{\lambda}$ in equality (23c).)

The input radial efficiency of DMU $C$ in technology $\mathcal{T}_{\text {NIRS }}$ is equal to 0.5 , and its input projection is $F$. 


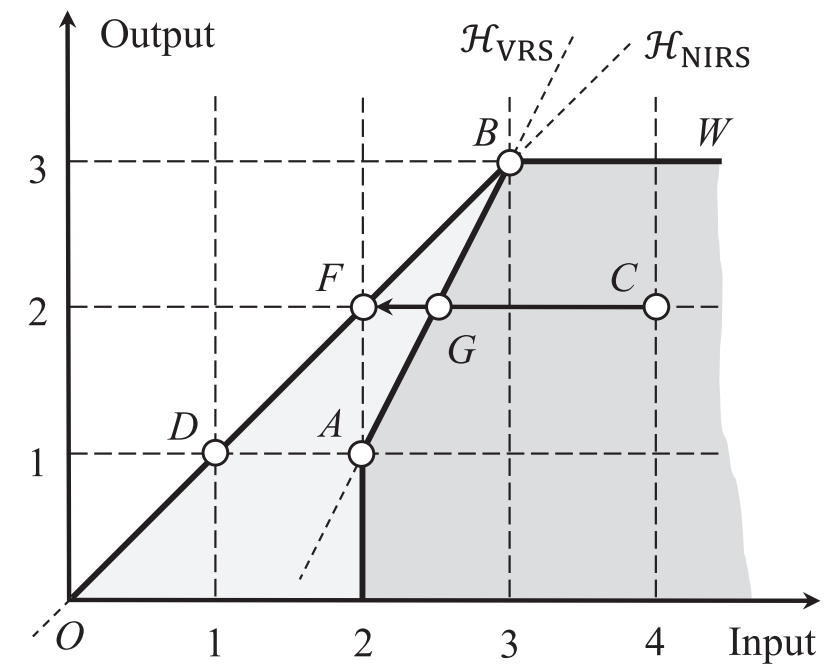

Fig. 1 Technologies $\mathcal{T}_{\text {NIRS }}$ and $\mathcal{T}_{\text {VRS }}$

The multiplier program (24) has the unique optimal solution $\left(u^{*}, v^{*}, u_{0}^{*}\right)=(0.25,0.25,0)$. In line with Proposition 1 , it defines the supporting hyperplane $\mathcal{H}_{\mathrm{NIRS}}$ to technology $\mathcal{T}_{\text {NIRS }}$ at the input projection $F$. This hyperplane satisfies the equation $0.25 Y-0.25 X=0$ and includes the line $O B$ as a subset. All DMUs in technology $\mathcal{T}_{\text {NIRS }}$ are located to one side of $\mathcal{H}_{\text {NIRS, }}$ more precisely, for any DMU $(X, Y) \in \mathcal{T}_{\text {NIRS }}$, we have $0.25 Y-0.25 X \leq 0$.

Similarly, the input radial efficiency of DMU $C$ in technology $\mathcal{T}_{\text {VRS }}$ is equal to 0.625 . The multiplier VRS model for this DMU has the unique optimal solution $\left(\hat{u}, \hat{v}, \hat{u}_{0}\right)=(0.125,0.25,0.375)$ which defines the supporting hyperplane $\mathcal{H}_{\mathrm{VRS}}$ to technology $\mathcal{T}_{\mathrm{VRS}}$ at the input projection $G$. This hyperplane satisfies the equation $0.125 Y$ $-0.25 X+0.375=0$ and includes the line segment $A B$ as a subset. All DMUs in technology $\mathcal{T}_{\text {VRS }}$ are located to one side of the hyperplane $\mathcal{H}_{\mathrm{VRS}}$, i.e., for any DMU $(X, Y) \in \mathcal{T}_{\text {VRS }}$, we have $0.125 Y-0.25 X+0.375 \leq 0$.

\subsection{Benchmarking efficiency ratio against the observed DMUs only}

Consider the following question: what are the best input and output weights $v \geq 0$ and $u \geq 0$, and the sign-free constant $u_{0}$ that maximize the efficiency ratio $\left(Y^{\top} u+u_{0}\right) / X^{\top} v$ of $\mathrm{DMU}_{o}$ $=C$ in relation to the maximum of such ratios of all observed DMUs? Note that this question does not refer to any technology. We are simply interested in the triplet $(u, v$, $u_{0}$ ), where $u \geq 0$ and $v \geq 0$, which is the most favorable for DMU $C$ in comparison to the observed DMUs. This question does not require that we assume any production technology.

Note that the above question is in line with the original motivation of the CRS model by Charnes et al. (1978). This model is first introduced in the linear fractional multiplier
Table 2 Efficiency ratios for different solutions

\begin{tabular}{lll}
\hline DMU & $\begin{array}{l}\text { Optimal in NIRS model: } \\
u^{*}=0.25, v^{*}=0.25, \\
u_{0}^{*}=0\end{array}$ & $\begin{array}{l}\text { Optimal in VRS model: } \\
\hat{u}=0.125, \hat{v}=0.25, \\
\hat{u}_{0}=0.375\end{array}$ \\
\hline$A$ & 0.5 & 1 \\
$B$ & 1 & 1 \\
$C$ & 0.5 & 0.625 \\
$D$ & 1 & 2
\end{tabular}

form, without a reference to any particular technology. It is presented as a model that maximizes the efficiency ratio $Y^{\top} u / X^{\top} v$ of $\mathrm{DMU}_{o}$ when it is benchmarked against the finite set of observed DMUs.

For ease of reference, Table 2 shows the efficiency ratios $\left(Y^{\top} u+u_{0}\right) / X^{\top} v$ of different DMUs (including the unobserved DMU $D$ ) evaluated at the optimal solutions $\left(u^{*}, v^{*}, u_{0}^{*}\right)$ and $\left(\hat{u}, \hat{v}, \hat{u}_{0}\right)$ of the NIRS and VRS multiplier models that assess the input radial efficiency of DMU $C$.

As noted in Remark 5, the answer to the above question is obtained by solving the standard input-oriented multiplier VRS model. In our example, this is model (24) in which the condition $u_{0} \leq 0$ is removed and the variable $u_{0}$ is declared sign free. The solution $\left(\hat{u}, \hat{v}, \hat{u}_{0}\right)$ optimal in the VRS model shows DMU $C$ in the best light in comparison the set of observed DMUs only. As seen from Table 2, for this solution, the ratio of the efficiency ratio of DMU $C$ to the maximum of such ratios of all observed DMUs $A, B$ and $C$ (but not $D$ ) is equal to $0.625 / \max \{1,1,0.625\}=0.625$.

Note that the solution $\left(u^{*}, v^{*}, u_{0}^{*}\right)$ obtained in the NIRS model is worse in this respect because $0.5 / \max \{0.5,1,0.5\}=0.5<0.625$. Therefore, the optimal solution of the NIRS model is not the most favorable for DMU $C$ when it is benchmarked against the set of observed DMUs. Note that the solution $\left(u^{*}, v^{*}, u_{0}^{*}\right)$ is also feasible in the VRS model, but this is not required and is unrelated to the stated question.

\subsection{Benchmarking efficiency ratio against the NIRS technology}

We now consider a question the answer to which depends on the assumed technology: what is the most favorable triplet $\left(u, v, u_{0}\right)$, where $v \geq 0, u \geq 0$ and $u_{0}$ is sign-free, that maximize the efficiency ratio $\left(Y^{\top} u+u_{0}\right) / X^{\top} v$ of $\mathrm{DMU}_{o}=C$ in relation to the maximum of such ratios of all DMUs in technology $\mathcal{T}_{\text {NIRS }}$ ?

In contrast to the question answered in Section 8.3, we now have to compare the efficiency ratio of $\mathrm{DMU}_{o}$ with the efficiency ratios of all DMUs in technology $\mathcal{T}_{\text {NIRS. For }}$ example, this includes DMU $D$ which is not observed but belongs to the technology by the assumption of NIRS. However, exactly as in Section 8.3, the only condition on 
the vectors $v$ and $u$ is that they should be nonnegative. In particular, the triplet $\left(u, v, u_{0}\right)$ is not required to satisfy any other conditions of the multiplier NIRS program (24), which is simply a normalized form of the corresponding maximin program (20)—see a related discussion in Section 5.

It is clear that any most favorable triplet $\left(u, v, u_{0}\right)$ in the described setting is an optimal solution of the maximin program (20), stated for $\mathrm{DMU}_{o}=C$, where we take $\mathcal{T}=\mathcal{T}_{\text {NIRS }}$. By Theorem 4 , and because in our example $u_{0}^{*}=w^{*}$, the optimal solution $\left(u^{*}, v^{*}, u_{0}^{*}\right)$ of the standard multiplier NIRS program (24) is also optimal in the maximin program (20). Therefore, $\left(u^{*}, v^{*}, u_{0}^{*}\right)$ is the most favorable triplet for DMU $C$ when it is benchmarked against the entire technology $\mathcal{T}_{\text {NIRS. }}$.

Furthermore, by Theorem 4 , the optimal value $\theta^{*}=0.5$ of program (24) is equal to the optimal value $\varphi_{3}^{*}$ of the corresponding program (20) and represents the maximal ratio of the efficiency ratio of DMU $C$ to the maximum of similar efficiency ratios calculated for all DMUs in technology $\mathcal{T}_{\text {NIRS. }}$.

Now consider the optimal solution $\left(\hat{u}, \hat{v}, \hat{u}_{0}\right)$ of the VRS multiplier model. It is clear that this solution is feasible in the maximin program (20) stated for $\mathrm{DMU}_{o}=$

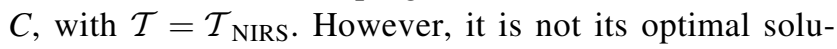
tion and is, therefore, not the most favorable for DMU $C$ when it is compared to the entire technology $\mathcal{T}_{\text {NIRS }}$, in the sense of maximizing its efficiency ratio. Indeed, to see this, it suffices to find a DMU $\left(X^{\prime}, Y^{\prime}\right)$ in technology $\mathcal{T}_{\text {NIRS }}$ such that the ratio of the efficiency ratios of DMUs $C$ and $\left(X^{\prime}, Y^{\prime}\right)$, calculated for $\left(\hat{u}, \hat{v}, \hat{u}_{0}\right)$, is strictly less than 0.5 .

As an example of the required DMU $\left(X^{\prime}, Y^{\prime}\right)$, consider DMU $D$. As shown in Table 2, for the optimal solution $\left(\hat{u}, \hat{v}, \hat{u}_{0}\right)$ of the VRS multiplier model, the efficiency ratio of DMU $C$ is equal to 0.625 , and the same ratio of DMU $D$ is equal to 2 . The ratio of these efficiency ratios is equal to $0.625 / 2=0.3125$, which is lower than the optimal value 0.5 achieved at the solution $\left(u^{*}, v^{*}, u_{0}^{*}\right)$. Therefore, the optimal solution $\left(\hat{u}, \hat{v}, \hat{u}_{0}\right)$ of the VRS multiplier model is not the most favorable for DMU $C$ when it is compared to all DMUs in technology $\mathcal{T}_{\text {NIRS. }}$.

Remark 6 It may be noted that the optimal solution $\left(\hat{u}, \hat{v}, \hat{u}_{0}\right)$ of the VRS multiplier model, as shown in Table 2, does not satisfy the constraints of the multiplier NIRS model (24), for example, because $\hat{u}_{0}=0.375>0$. It is also clear from Fig. 1 that the corresponding supporting hyperplane $\mathcal{H}_{\text {VRS }}$ to the VRS technology is not a supporting hyperplane to the NIRS technology. However, these observations are unrelated to the question asked at the beginning of this section. The only condition imposed by the question is the nonnegativity of the weights $u$ and $v$.
Table 3 Profits for different solutions

\begin{tabular}{lll}
\hline DMU & $\begin{array}{l}\text { Optimal in NIRS model: } \\
u^{*}=0.25, v^{*}=0.25, \\
u_{0}^{*}=0\end{array}$ & $\begin{array}{l}\text { Optimal in VRS model: } \\
\hat{u}=0.125, \hat{v}=0.25, \\
\hat{u}_{0}=0.375\end{array}$ \\
\hline$A$ & -0.25 & -0.375 \\
$B$ & 0 & -0.375 \\
$C$ & -0.5 & -0.75 \\
$D$ & 0 & -0.125 \\
\hline
\end{tabular}

Therefore, any triplet $\left(u, v, u_{0}\right)$ that satisfies this condition is an eligible candidate for the maximization of the efficiency ratio of DMU $C$ by the maximin program (20). The optimal solution $\left(\hat{u}, \hat{v}, \hat{u}_{0}\right)$ of the VRS multiplier program is feasible in the maximin program (20) but, as shown, is not optimal. In contrast, by Theorem 4 , the optimal solution $\left(u^{*}, v^{*}, u_{0}^{*}\right)$ of the multiplier NIRS program (24) is also optimal in program (20), and provides the answer to the stated question.

\subsection{Benchmarking profit efficiency against the NIRS technology}

Let us reinterpret the same example in terms of profit efficiency. Let $v \geq 0$ and $u \geq 0$ represent the input and output prices. The second column of Table 3 shows the profit of the observed DMUs $A, B$ and $C$, and the unobserved DMU $D$, calculated for the input and output prices $v^{*}=u^{*}=0.25$ taken from the optimal solution $\left(u^{*}, v^{*}, u_{0}^{*}\right)$ of the multiplier NIRS model (24). For example, the profit of DMU $C$ is equal to $2 \times 0.25-4 \times 0.25=-0.5$. The last column shows the profit of these DMUs calculated for the prices $\hat{v}=0.25$ and $\hat{u}=0.125$ taken from the optimal solution $\left(\hat{u}, \hat{v}, \hat{u}_{0}\right)$ of the VRS multiplier model.

Consider the following question: what input and output prices $v$ and $u$ minimize the difference between the maximum profit across all (observed and unobserved) DMUs in technology $\mathcal{T}_{\text {NIRS }}$ and the profit of DMU $C$, under the normalizing condition that the cost of the input bundle of DMU $C$ is equal to 1 (i.e., $4 v=1$ in our example)? In other words, what input and output prices are the most favorable for DMU $C$ in the sense of profit efficiency evaluated in comparison to the entire technology?

The general answer to this question was obtained in Section 4.1. In the context of our example, such most favorable prices $v^{*}$ and $u^{*}$ are taken from the optimal solution $\left(u^{*}, v^{*}, u_{0}^{*}\right)$ of the multiplier NIRS program (24), i.e., $v^{*}=u^{*}=0.25$. As shown in Section 4.1, for these prices, the maximum profit achievable across all DMUs in technology $\mathcal{T}_{\text {NIRS }}$ is equal to $-u_{0}^{*}=0$. (Note that this maximum is consistent with the profits of all four DMUs shown in the second column of Table 3, although of course 
these DMUs do not represent the whole technology $\mathcal{T}_{\text {NIRS.) }}$ The difference between this maximum profit and the profit of DMU $C$ is calculated as $0-(-0.5)=0.5$.

It is easy to show that the input and output prices $\hat{v}=$ 0.25 and $\hat{u}=0.125$ taken from the optimal solution $\left(\hat{u}, \hat{v}, \hat{u}_{0}\right)$ of the VRS model do not maximize the profit efficiency of DMU $C$ benchmarked against the entire technology $\mathcal{T}_{\text {NIRS. }}$. Note that such prices satisfy the conditions stated in the question about profit efficiency maximization: both prices are positive and the cost of the input bundle of DMU $C$ is equal to 1.) Indeed, it suffices to identify a DMU $\left(X^{\prime}, Y^{\prime}\right) \in \mathcal{T}_{\text {NIRS }}$ such that the difference between the profits of DMUs $\left(X^{\prime}, Y^{\prime}\right)$ and $C$ for the prices $\hat{v}$ and $\hat{u}$ is greater than 0.5 .

As an example of DMU $\left(X^{\prime}, Y^{\prime}\right)$, consider DMU D. As follows from the last column of Table 3 , the difference between the profits of DMUs $D$ and $C$ is equal to $-0.125-$ $(-0.75)=0.625>0.5$. Therefore, the optimal prices $\hat{v}$ and $\hat{u}$ of the multiplier VRS model do not maximize the profit efficiency of DMU $C$ when it is benchmarked against the entire technology.

\subsection{Benchmarking profit efficiency against the observed DMUs}

Now suppose that we want to identify the input and output prices that maximize the profit of DMU $C$ in comparison to the maximum profit among the three observed DMUs only. Similar to the question considered in Section 8.3, the answer to this new question does not depend on the assumed technology.

The prices that answer the stated question are the ones given by the optimal solution $\left(\hat{u}, \hat{v}, \hat{u}_{0}\right)$ of the VRS model. (To prove this, we need to repeat the discussion of profit maximization in Section 4.1, with the technology $\mathcal{T}$ replaced by the set of observed DMUs $\left(X_{j}, Y_{j}\right), j=1, \ldots, n$, and program (7) replaced by program (15).) For the optimal solution $\left(\hat{u}, \hat{v}, \hat{u}_{0}\right)$, the difference between the maximum profit and the profit of DMU $C$ is equal to $-0.375-$ $(-0.75)=0.375$. This represents a smaller shortfall compared to the shortfall of 0.5 in the case of benchmarking $\mathrm{DMU}_{o}$ against the entire NIRS technology considered in Section 8.5.

Note that, if we used the prices $v^{*}=u^{*}=0.25$ identified by the multiplier NIRS program (24), the profit of DMU $C$ would be equal to -0.5 and the maximum profit among the observed DMUs would be equal to 0 (the profit of DMU $B$ ), with the difference equal to $0-(-0.5)=0.5$, which is greater than the difference 0.375 achieved at the optimal prices in the VRS model. Therefore, the optimal prices obtained by solving the multiplier NIRS program (24) do not maximize the profit efficiency of $\mathrm{DMU}_{o}$ when it is benchmarked against the observed DMUs only.

\subsection{Summary and discussion}

This numerical example illustrates the theoretical results obtained in the previous sections. It shows that it would be incorrect to interpret the optimal solution $\left(u^{*}, v^{*}, u_{0}^{*}\right)$ of the multiplier NIRS model as being the most favorable for DMU $C$ when it is compared to the observed DMUs only, both in the context of maximizing its efficiency ratio and in the context of profit efficiency maximization. Instead, in line with the theory, this solution is the most favorable for DMU $C$ when it is benchmarked against the entire technology $\mathcal{T}_{\text {NIRS. }}$.

We can also look at our results from a different perspective. The difference between the input radial efficiencies of DMU $C$ in the multiplier VRS and NIRS models, equal to $0.625-0.5=0.125$, or the ratio of such efficiencies, may be viewed as a measure of the difference between benchmarking DMU $C$ against the observed DMUs only (equivalent to solving the VRS model) and against the entire NIRS technology. Such measure represents the value of the assumption of non-increasing returns to scale, or, alternatively, the value of unobserved DMUs included in the NIRS technology, in addition to the observed DMUs.

Remark 7 In some applications of DEA, multiplier models are used for the benchmarking of DMUs against the set of observed DMUs only, without assuming any production technology or considering the meaning of the dual envelopment model-see the discussion in Cook et al. (2014). The standard multiplier CRS and VRS models in this approach are often modified in order to incorporate additional information relevant for the benchmarking purposes. Examples include traditional use of weight restrictions (Allen et al. 1997) or incorporation of a particular mechanism for the allocation of shared inputs and outputs between component production processes, by using additional variable allocation factors in the multiplier model (Cook et al. 2000). ${ }^{13}$ Some of these modified multiplier models cannot easily be stated in the standard form (6) and their dual programs do not reveal a clear production technology in the same way as the envelopment model (5).

The benchmarking approaches based entirely on the multiplier DEA models have important limitations. Because no technology is assumed, the traditional interpretation of efficiency as the ultimate feasible (possible) improvement factor for the $\mathrm{DMU}_{o}$ under the assessment becomes unsubstantiated. Because the production frontier is

\footnotetext{
13 The alternative approaches of Podinovski (2004b) and Podinovski et al. (2018) allow the specification and interpretation of weight restrictions and multi-component processes in terms of the underlying production technology $\mathcal{T}$ and both envelopment and multiplier models based on it.
} 
undefined, the various scale characteristics such as scale efficiency, most productive scale size and returns to scale are not defined rigorously and can be interpreted only heuristically. Finally, the notions of productivity and productivity change remain undefined.

In contrast with such benchmarking approaches that are based entirely on the multiplier model, in this paper, we start by assuming a production technology $\mathcal{T}$ stated by conditions (4). This means that all DMUs in technology $\mathcal{T}$ are considered producible and, together with the observed DMUs, are intended to be used for the benchmarking of the $\mathrm{DMU}_{o}$. If we still want to evaluate the efficiency of $\mathrm{DMU}_{o}$ against the set of observed DMUs only, we can do so by solving the standard CRS or VRS model, but not the multiplier model (6) based on technology $\mathcal{T}$, which, as shown, would benchmark $\mathrm{DMU}_{o}$ against all, observed and unobserved, DMUs in $\mathcal{T}$. However, apart from some cases of special interest (for example, in order to compare the efficiency scores in technologies $\mathcal{T}_{\text {VRS }}$ and $\mathcal{T}$ ), assuming a particular technology $\mathcal{T}$ (different from the standard VRS or CRS technologies) and, at the same time, benchmarking $\mathrm{DMU}_{o}$ against the set of observed DMUs would appear to be two inconsistent tasks.

\section{Conclusion}

A clear and rigorous interpretation of optimal input and output weights is important for explaining the meaning of multiplier DEA models and the efficiency of DMUs obtained from them. According to the standard interpretation given by Charnes et al. (1978), any optimal weights of the multiplier CRS model are the most favorable for the $\mathrm{DMU}_{o}$ under the assessment if it is benchmarked against the set of all observed DMUs. Such weights maximize the ratio of the total weighted output to the total weighted input of $\mathrm{DMU}_{o}$ in relation to the maximum of similar ratios taken across all observed DMUs. This ratio may be bounded above by the value of 1 , as in the linear fractional model, or not bounded, as in the maximin DEA model. The VRS model allows a similar interpretation and also includes an additional sign-free variable interpretable in terms of the type of RTS exhibited by the radial projection of $\mathrm{DMU}_{o}$.

The CRS and VRS technologies are examples of a very large class of polyhedral technologies. The multiplier models based on such technologies often have a complex structure which makes the interpretation of their optimal solutions problematic. As highlighted in our paper, the conventional interpretation of optimal solutions as being the most favorable for $\mathrm{DMU}_{o}$, which is often used heuristically by analogy to the CRS and VRS models as the default interpretation, is generally incorrect in the class of polyhedral technologies.
In this paper we obtain new results that give us a unifying interpretation of the multiplier models and their optimal solutions. We show that the optimal solutions of the multiplier model based on any polyhedral technology are the most favorable for $\mathrm{DMU}_{o}$ when it is benchmarked against all DMUs in the entire technology, but not necessarily in comparison to the set of observed DMUs as the conventional interpretation would imply. We explore the precise meaning of this interpretation by transforming the multiplier DEA model to its alternative linear, linear fractional and maximin forms which have a transparent structure.

To summarize these results, consider the input-oriented multiplier model (6) based on any polyhedral technology $\mathcal{T}$ stated in the form (4). Let $\left(u^{*}, v^{*}, w^{*}\right)$ be any of its optimal solutions. Further define $u_{0}^{*}=U_{o}^{\top} w^{*}$, where $U_{o}$ is the vector on the right-hand side of the parameter conditions (4c). As proved in this paper, the following two interpretations are always valid.

Interpretation 1 The triplet $\left(u^{*}, v^{*}, u_{0}^{*}\right)=\left(u^{*}, v^{*}, U_{o}^{\top} w^{*}\right)$ maximizes the efficiency ratio $\left(Y_{o}^{\top} u+u_{0}\right) / X_{o}^{\top} v$ of $\mathrm{DMU}_{o}$ relative to (divided by) the maximum of efficiency ratios of all DMUs in the entire technology $\mathcal{T}$. This maximization is performed over the set of all triplets $\left(u, v, u_{0}\right)$ under the single condition $u, v \geq 0$ and treating $u_{0}$ as a sign-free variable.

This interpretation remains true if the set of feasible triplets $\left(u, v, u_{0}\right)$ is additionally limited by the requirement that the efficiency ratios of all DMUs in technology $\mathcal{T}$ are bounded above by 1 , as in the linear fractional model (18).

A special case arises if the parameter conditions (4c) in the statement of technology $\mathcal{T}$ are not specified (as, for example, in the standard CRS technology) or if the vector $U_{o}$ in these parameter conditions is a zero vector. In this case, $\mathcal{T}$ is a cone technology and the scalar $u_{0}$ is either omitted or is equal to zero. In both cases, the optimal weights $u^{*}$ and $v^{*}$ maximize the efficiency ratio $Y_{o}^{\top} u / X_{o}^{\top} v$ of $\mathrm{DMU}_{o}$ relative to the maximum of efficiency ratios of all DMUs in the entire technology $\mathcal{T}$. This interpretation is true if the maximization is performed over all vectors $u$ and $v$, subject to the single condition $u, v \geq 0$, or under the additional requirement that the efficiency ratios of all DMUs in technology $\mathcal{T}$ are bounded above by 1 , as in the linear fractional model (21).

Interpretation 2 Let $v$ and $u$ be viewed as the vectors of input and output prices. Then the components of any optimal solution $\left(u^{*}, v^{*}, w^{*}\right)$ of the multiplier model (6) allow the following interpretation. The vectors of prices $v^{*}$ and $u^{*}$ minimize the difference between the maximum profit achieved in technology $\mathcal{T}$ and the profit made by $\mathrm{DMU}_{o}$, subject to the condition that the cost of the input bundle $X_{o}$ is normalized by 1 (i.e., $X_{o}^{\top} v=1$ ). In other words, the 
optimal prices $v^{*}$ and $u^{*}$ maximize the profit efficiency of $\mathrm{DMU}_{o}$ when it is benchmarked against all DMUs in technology $\mathcal{T}$. Furthermore, for the vectors of prices $v^{*}$ and $u^{*}$, the maximum profit among all DMUs in technology $\mathcal{T}$ is equal to $-u_{0}^{*}=-U_{o}^{\top} w^{*}$.

As noted in Interpretation 1 , in the special case of cone technologies in which vector $U_{o}$ is either omitted or is a zero vector, the scalar $u_{0}$ is also either omitted or is equal to zero. In this case the maximum profit in the entire technology $\mathcal{T}$ is always equal to zero (which is consistent with the assumption of CRS), and the optimal vectors of prices $v^{*}$ and $u^{*}$ simply maximize the (nonpositive) profit made by $\mathrm{DMU}_{o}$, among all nonnegative $v$ and $u$.

For the multiplier CRS and VRS models of Charnes et al. (1978) and Banker et al. (1984), both the conventional interpretation of their optimal solutions and the new Interpretations 1 and 2 are correct. In the case of CRS and VRS technologies, benchmarking $\mathrm{DMU}_{o}$ against the set of observed DMUs and against the entire technology is the same task.

In contrast, for an arbitrary polyhedral technology, only the new Interpretations 1 and 2 are generally correct. This is because, as demonstrated by an example in Section 8 , the optimal weights that are the most favorable for $\mathrm{DMU}_{o}$ when it is compared to the observed DMUs are not necessarily the most favorable when it is benchmarked against the entire technology, and vice versa.

Interestingly, the interpretations obtained in this paper also remove the apparent disparity between the conventional interpretations of the envelopment and multiplier models. Namely, the envelopment DEA model benchmarks $\mathrm{DMU}_{o}$ against the entire technology. (For example, in the input-oriented envelopment model (5), the input improvement factor $\theta$ is minimized as long as the improved DMU $\left(\theta X_{o}, Y_{o}\right)$ remains in technology $\mathcal{T}$.) However, the multiplier DEA model (e.g., the CRS model as originally motivated by Charnes et al. (1978)) is traditionally explained as benchmarking $\mathrm{DMU}_{o}$ against the observed DMUs only. Our results show that the multiplier model always benchmarks $\mathrm{DMU}_{o}$ against the entire technology, exactly as the envelopment model. (In the case of VRS and CRS technologies, it also benchmarks against the set of observed DMUs, but for an arbitrary technology, this is generally not so.)

Another result obtained in our paper concerns the mathematical statement of the supporting hyperplanes to the technology at the radial projection of $\mathrm{DMU}_{o}$ under the assessment. Because any polyhedral technology is a convex set, there always exists a supporting hyperplane at the projection point of any DMU. In the case of conventional CRS and VRS technologies, such hyperplanes are easily identified by the optimal solutions to the multiplier CRS and VRS models (Banker et al. 1984). However, in the general case of polyhedral technology, because of the often complex structure of constraints and solutions of the multiplier model, the mathematical statement of the supporting hyperplanes is not obvious. In our paper we overcome this problem and obtain an explicit equation of the supporting hyperplane corresponding to any optimal solution of the multiplier model. This new result is useful for the returnsto-scale characterization of efficient frontiers of arbitrary polyhedral technologies and clarifies the meaning of the previously published results in this field (Podinovski et al. 2016).

The approach developed in this paper should also be applicable to models employing nonradial efficiency measures, for example, the nonparametric directional distance function models (Chambers et al. 1998) and slack-based measures (Tone 2001). Another potential research avenue involves models of cost and revenue efficiency (Färe et al. 1994). Detailed exploration of the multiplier forms of these models within the suggested framework and the interpretation of optimal solutions arising from this approach is left outside the scope of our paper for future research.

\section{Compliance with ethical standards}

Conflict of interest The authors declare no competing interests.

Publisher's note Springer Nature remains neutral with regard to jurisdictional claims in published maps and institutional affiliations.

Open Access This article is licensed under a Creative Commons Attribution 4.0 International License, which permits use, sharing, adaptation, distribution and reproduction in any medium or format, as long as you give appropriate credit to the original author(s) and the source, provide a link to the Creative Commons license, and indicate if changes were made. The images or other third party material in this article are included in the article's Creative Commons license, unless indicated otherwise in a credit line to the material. If material is not included in the article's Creative Commons license and your intended use is not permitted by statutory regulation or exceeds the permitted use, you will need to obtain permission directly from the copyright holder. To view a copy of this license, visit http://creativecommons. org/licenses/by/4.0/.

\section{Appendix A: Proofs}

The proofs given below extend the idea of the proofs given by Podinovski et al. (2016) for a special case of technology $\mathcal{T}$ to the case of an arbitrary polyhedral technology.

Lemma 1 The maximum in program (7) is attained and $\theta^{*}=\varphi_{1}^{*}$.

Proof of Lemma 1. Because $\mathcal{T}$ is a polyhedral set, it is generated by a finite number of points $\left(A_{k}, B_{k}\right) \in \mathbb{R}^{m+s}$, $k=1, \ldots, \tilde{k}, \quad$ and directions $\left(A_{k}, B_{k}\right) \in \mathbb{R}^{m+s}, \quad k=$ $\tilde{k}+1, \ldots, k^{\prime}$ (Rockafellar 1970, Theorem 19.1). More precisely, $\mathcal{T}$ consists of all DMUs $(X, Y)$ for which there 
exists a vector $\mu \in \mathbb{R}_{+}^{k^{\prime}}$ such that:

$X=\sum_{k=1}^{k^{\prime}} \mu_{k} A_{k}, \quad Y=\sum_{k=1}^{k^{\prime}} \mu_{k} B_{k}, \sum_{k=1}^{\tilde{k}} \mu_{k}=1$.

Using (25), we restate program (5) as follows:

$\theta^{*}=\min \theta$

subject to $\theta X_{o}-\sum_{k=1}^{k^{\prime}} \mu_{k} A_{k}=0$,

$\sum_{k=1}^{k^{\prime}} \mu_{k} B_{k}=Y_{o}$

$\sum_{k=1}^{\tilde{k}} \mu_{k}=1$,

$\mu \geq 0, \theta$ sign free.

Because technology $\mathcal{T}$ is freely disposable, we can replace the equality signs in (26b) and (26c) by the " $\geq$ " inequalities. Consider the dual to (26):

$\theta^{*}=\max Y_{o}^{\top} u+u_{0}$

subject to $X_{o}^{\top} v=1$,

$B_{k}^{\top} u-A_{k}^{\top} v+u_{0} \leq 0, k=1, \ldots, \tilde{k}$,

$B_{k}^{\top} u-A_{k}^{\top} v \leq 0, k=\tilde{k}+1, \ldots, k^{\prime}$,

$u, v \geq 0, u_{0}$ sign free.

Any DMU $(X, Y) \in \mathcal{T}$ satisfies equalities (25) with some vector $\mu \geq 0$. Multiply constraints $k=1, \ldots, k^{\prime}$ in (27c) and (27d) by $\mu_{k} \geq 0$ and add together. We have

$Y^{\top} u-X^{\top} v+u_{0} \leq 0, \forall(X, Y) \in \mathcal{T}$

We have proved that constraints (27c) and (27d) imply (28). We can therefore incorporate constraint (28) in program (27) without affecting its feasible region. Furthermore, because $\left(A_{k}, B_{k}\right) \in \mathcal{T}$, for all $k=1, \ldots, \tilde{k}$, in the resulting program constraints (27c) now follow from (28) and can be removed as redundant.

Let us prove that constraints (27d) also follow from (28). Consider any $k=\tilde{k}+1, \ldots, k^{\prime}$. By (25), we have $(X, Y)=\left(A_{1}+\mu_{k} A_{k}, B_{1}+\mu_{k} B_{k}\right) \in \mathcal{T}, \forall \mu_{k}>0$. Then (28) implies

$\left(B_{1}+\mu_{k} B_{k}\right)^{\top} u-\left(A_{1}+\mu_{k} A_{k}\right)^{\top} v+u_{0} \leq 0, \forall \mu_{k}>0$.
Suppose that the triplet $\left(u, v, u_{0}\right)$ satisfies inequalities (28) and, as proved, (29). Divide both sides of inequality (29) by $\mu_{k}>0$. Taking $\mu_{k} \rightarrow+\infty$, at the limit we obtain inequality $k$ in constraint $(27 \mathrm{~d})$. We have proved that constraint (28) implies constraints $(27 \mathrm{~d})$. Removing the latter as redundant, we obtain program (14).

Proof of Theorem 1. Let $(u, v, w)$ be feasible in (6). Let us prove that $\left(u, v, u_{0}\right)$ is feasible in (7), where $u_{0}$ is as defined by (8). It suffices to prove that condition (7c) is satisfied. Any $\operatorname{DMU}(X, Y) \in \mathcal{T}$ can be stated in the form (4) with some $\hat{\lambda}, S_{X}, S_{Y} \geq 0$. Multiply each inequality $j=1$, $\ldots, q$ in (6c) by the corresponding $\hat{\lambda}_{j}$ and add together. Noting that $S_{X}, S_{Y} \geq 0$ and taking into account (4c), we have

$Y^{\top} u-X^{\top} v+U_{o}^{\top} w \leq 0$

By definition of $u_{0}$, the latter inequality is the same as (7c). Therefore, $\left(u, v, u_{0}\right)$ is feasible in (7). Finally, let $\left(u^{*}, v^{*}, w^{*}\right)$ be an optimal solution of (6). Then, as proved, $\left(u^{*}, v^{*}, u_{0}^{*}\right)$ is feasible in (7). From (6a), we have $Y_{o}^{\top} u^{*}+u_{0}^{*}=\theta^{*}$. By Lemma $1, \theta^{*}=\varphi_{1}^{*}$. Therefore, $\left(u^{*}, v^{*}, u_{0}^{*}\right)$ is optimal in program (7).

Proof of Proposition 1. Because $\left(u^{*}, v^{*}, u_{0}^{*}\right)$ is optimal in (7), we have $Y_{o}^{\top} u^{*}+u_{0}^{*}=\theta^{*}$. Multiplying (7b) by $\theta^{*}$, we have $\theta^{*} X_{o}^{\top} v^{*}=\theta^{*}$. Then $Y_{o}^{\top} u^{*}-\theta^{*} X_{o}^{\top} v^{*}+u_{0}^{*}=0$. Therefore, the projection $\left(\theta^{*} X_{o}, Y_{o}\right)$ satisfies (10). By (7c), we also have $Y^{\top} u^{*}-X^{\top} v^{*}+u_{0}^{*} \leq 0$, for all $(X, Y) \in \mathcal{T}$.

Proof of Theorem 2 and Proposition 2. These results are obtained as special cases of Theorem 1 and Proposition 1 , respectively, by noting that the vector $U_{o}$ in (8) is a zero vector.

Lemma 2 The feasible region of program (7) remains the same if constraints $(7 \mathrm{c})$ are changed to

$Y^{\top} u-X^{\top} v+u_{0} \leq 0, \forall(X, Y) \in \mathcal{T}: X^{\top} v>0$

i.e., if constraints (7c) are required only for the DMUs $(X, Y) \in \mathcal{T}$ such that $X^{\top} v>0$.

Proof of Lemma 2. It suffices to prove that, if solution $\left(u, v, u_{0}\right)$ satisfies constraints (7b), (7d) and (30), then it also satisfies (7c). Let $(\tilde{X}, \tilde{Y}) \in \mathcal{T}$. If $\tilde{X}^{\top} v>0$ then (7c) follows from (30). If $\tilde{X}^{\top} v=0$, define DMUs $\left(X^{k}, Y^{k}\right), k=1,2, \ldots$, as follows:

$\left(X^{k}, Y^{k}\right)=(1-1 / k)(\tilde{X}, \tilde{Y})+(1 / k)\left(X_{o}, Y_{o}\right)$

Technology $\mathcal{T}$ is a polyhedral set. Therefore, $\mathcal{T}$ is a convex set and $\left(X^{k}, Y^{k}\right) \in \mathcal{T}, \forall k$. Taking into account (7b) and (31), we have $\left(X^{k}\right)^{\top} v>0, \forall k$. By (30), $\left(Y^{k}\right)^{\top} u-\left(X^{k}\right)^{\top} v+u_{0} \leq 0, \forall k$. Taking $k \rightarrow+\infty$, at the limit we have $\tilde{Y}^{\top} u-\tilde{X}^{\top} v+u_{0} \leq 0$, which proves $(7 \mathrm{c})$. 
Proof of Theorem 3. Define the feasible region of program (18) as the set of all triplets $\left(u, v, u_{0}\right)$ such that 1$)$ $\left.X_{o}^{\top} v>0,2\right)$ constraints (18b) are true for all DMUs $(X, Y) \in$ $\mathcal{T}$ such that $X^{\top} v>0$, and 3) conditions (18c) are true. By Theorem 1, $\left(u^{*}, v^{*}, u_{0}^{*}\right)$ is optimal in (7) and $\theta^{*}=\varphi_{1}^{*}$. Furthermore, $\left(u^{*}, v^{*}, u_{0}^{*}\right)$ is feasible in (18). In particular, for all DMUs $(X, Y) \in \mathcal{T}$ such that $X^{\top} v^{*}>0$, the inequalities (7c) imply (18b).

Assume that $\left(u^{*}, v^{*}, u_{0}^{*}\right)$ is not an optimal solution of program (18). Then there exists a feasible solution $\left(u^{\prime}, v^{\prime}, u_{0}^{\prime}\right)$ to (18) for which $\left(Y_{o}^{\top} u^{\prime}+u_{0}^{\prime}\right) / X_{o}^{\top} v^{\prime}=\theta^{\prime}$ and $\theta^{\prime}>\varphi_{1}^{*}$. For any $\alpha>0$, solution $\left(\alpha u^{\prime}, \alpha v^{\prime}, \alpha u_{0}^{\prime}\right)$ is also feasible in (18). At this solution, the objective function (18a) is equal to $\left(Y_{o}^{\top} \alpha u^{\prime}+\alpha u_{0}^{\prime}\right) /\left(X_{o}^{\top} \alpha v^{\prime}\right)=\theta^{\prime}$. Define $\alpha^{\prime}$ so that $X_{o}^{\top} \alpha^{\prime} v^{\prime}=1$, i.e., let $\alpha^{\prime}=1 /\left(X_{o}^{\top} v^{\prime}\right)$. Note that $\left(\alpha^{\prime} u^{\prime}, \alpha^{\prime} v^{\prime}, \alpha u_{0}^{\prime}\right)$ is feasible in (18). To see this, note that constraints $(18 \mathrm{~b})$ are satisfied for all $(X, Y) \in \mathcal{T}$ such that $X^{\top} v>0$, and conditions (30) are proved. By Lemma 2, this implies (18c). The objective function (18a) at $\left(\alpha^{\prime} u^{\prime}, \alpha^{\prime} v^{\prime}, \alpha u_{0}^{\prime}\right)$ is equal to $\theta^{\prime}>\varphi_{1}^{*}$ which is impossible because $\varphi_{1}^{*}$ is the optimal value of (18).

Proof of Proposition 3. Define $\left(u^{\prime}, v^{\prime}, u_{0}^{\prime}\right)=$ $\left(\alpha u^{*}, \alpha v^{*}, \alpha u_{0}^{*}\right)$, where $\alpha=1 /\left(X_{o}^{\top} v^{*}\right)>0$. Then $\left(u^{\prime}, v^{\prime}, u_{0}^{\prime}\right)$ is optimal in (18). Note that $\left(u^{\prime}, v^{\prime}, u_{0}^{\prime}\right)$ is also feasible in (7). Indeed, for any $(X, Y) \in \mathcal{T}$ such that $X^{\top} v^{\prime}>0$, the inequality (7c) follows from (18b). For any $(X, Y) \in \mathcal{T}$ such that $X^{\top} v^{\prime}=0$, the inequality (7c) follows from Lemma 2 . Because $\quad \varphi_{2}^{*}=\left(Y_{o}^{\top} u^{\prime}+u_{0}^{\prime}\right) /\left(X_{o}^{\top} v^{\prime}\right)=Y_{o}^{\top} u^{\prime}+u_{0}^{\prime} \quad$ and $\varphi_{2}^{*}=\varphi_{1}^{*},\left(u^{\prime}, v^{\prime}, u_{0}^{\prime}\right)$ is optimal in (7). By Proposition 1, we have $Y_{o}^{\top} u^{\prime}+u_{0}^{\prime}=\theta^{*} X_{o}^{\top} v^{\prime}$. Dividing this equality by $\alpha$, we have $Y_{o}^{\top} u^{*}+u_{0}^{*}=\theta^{*} X_{o}^{\top} v^{*}$.

Proof of Theorem 4. We first use the approach of Podinovski et al. (2016) and restate program (20) in a more precise form that explicitly disallows division by zero:

$$
\varphi_{3}^{*}=\max _{\left(u, v, u_{0}\right) \in \Omega}\left(\frac{\left(Y_{o}^{\top} u+u_{0}\right) / X_{o}^{\top} v}{\sup _{(X, Y) \in \mathcal{T}_{v}}\left\{\left(Y^{\top} u+u_{0}\right) / X^{\top} v\right\}}\right),
$$

where the set $\Omega$ is defined as follows:

$$
\Omega=\left\{\left(u, v, u_{0}\right) \mid u, v \geq 0, X_{o}^{\top} v>0, Y_{o}^{\top} u+u_{0}>0\right\}
$$

and the definition of the set $\mathcal{T}_{v}$ depends on the vector $v$ of the solution $\left(u, v, u_{0}\right) \in \Omega$ :

$$
\mathcal{T}_{v}=\left\{(X, Y) \in \mathcal{T} \mid X^{\top} v>0\right\}
$$

Note that $\left(u^{*}, v^{*}, u_{0}^{*}\right) \in \Omega$ and $\Omega \neq \varnothing$. Indeed, (6b) implies $\quad X_{o}^{\top} v^{*}=1>0, \quad$ and (6a) implies $Y_{o}^{\top} u^{*}+u_{0}^{*}=\theta^{*}>0$. Also, for any $\left(u, v, u_{0}\right) \in \Omega$, DMU $\left(X_{o}, Y_{o}\right) \in \mathcal{T}_{v}$. Therefore, for all $\left(u, v, u_{0}\right) \in \Omega$, the set $\mathcal{T}_{v}$ is not empty and the supremum in the denominator of (32) is strictly positive. If, for some triplet $\left(u, v, u_{0}\right) \in \Omega$, this supremum is unbounded (equal to $+\infty$ ), the objective function of program (32), i.e., the expression in the big parentheses on the right-hand side of (32), is formally assumed to be equal to zero. This possibility was illustrated in Example 3 in Podinovski et al. (2016).

By Proposition 3, the objective function of program (32) evaluated at $\left(u^{*}, v^{*}, u_{0}^{*}\right)$ is equal to $\theta^{*}$. Therefore, $\varphi_{3}^{*} \geq \theta^{*}$.

Suppose that $\left(u^{*}, v^{*}, u_{0}^{*}\right)$ is not an optimal solution of program (32). Then there exists a triplet $\left(u^{\prime}, v^{\prime}, u_{0}^{\prime}\right) \in \Omega$ such that

$$
\varphi\left(u^{\prime}, v^{\prime}, u_{0}^{\prime}\right)=\frac{\left(Y_{o}^{\top} u^{\prime}+u_{0}^{\prime}\right) / X_{o}^{\top} v^{\prime}}{\sup _{(X, Y) \in \mathcal{T}_{v^{\prime}}}\left\{\left(Y^{\top} u^{\prime}+u_{0}^{\prime}\right) / X^{\top} v^{\prime}\right\}}=\theta^{\prime}>\theta^{*}
$$

Clearly, for any $\alpha>0$, we have $\left(u^{\prime}, \alpha v^{\prime}, u_{0}^{\prime}\right) \in \Omega$ and $\varphi\left(u^{\prime}, \alpha v^{\prime}, u_{0}^{\prime}\right)=\varphi\left(u^{\prime}, v^{\prime}, u_{0}^{\prime}\right)=\theta^{\prime}$. Let

$\alpha^{\prime}=\sup _{(X, Y) \in \mathcal{T}_{v^{\prime}}}\left\{\left(Y^{\top} u^{\prime}+u_{0}^{\prime}\right) / X^{\top} v^{\prime}\right\}$

From (33), we have $0<\alpha^{\prime}<+\infty$. Consider the triplet $\left(\tilde{u}, \tilde{v}, \tilde{u}_{0}\right)=\left(u^{\prime}, \alpha^{\prime} v^{\prime}, u_{0}^{\prime}\right)$.

Note that $\sup _{(X, Y) \in \mathcal{T}_{\tilde{v}}}\left\{\left(Y^{\top} \tilde{u}+\tilde{u}_{0}\right) / X^{\top} \tilde{v}\right\}=1 \quad$ and $\varphi\left(\tilde{u}, \tilde{v}, \tilde{u}_{0}\right)=\left(Y_{o}^{\top} \tilde{u}+\tilde{u}_{0}\right) / X_{o}^{\top} \tilde{v}=\theta^{\prime}$. Therefore, $\left(\tilde{u}, \tilde{v}, \tilde{u}_{0}\right)$ is a feasible solution of program (18), and the objective function (18a) at this solution is equal to $\theta^{\prime}$. By (33), $\theta^{\prime}>\theta^{*}$. This is, however, impossible because, by Theorem $3, \theta^{*}$ is the optimal value of program (18).

Proof of Theorems 5, 6 and Proposition 4. All three results are special cases of Theorems 3 and 4, and Proposition 3 , respectively.

\section{Appendix B: Examples of interpretation for polyhedral technologies}

Below we consider multiplier models based on three different polyhedral technologies. We show how the suggested unifying interpretation of optimal solutions applies to these models.

\subsection{The hybrid returns-to-scale (HRS) technology}

Consider the HRS technology $\mathcal{T}_{\text {HRS }}$ introduced in Section 2. As shown, a direct interpretation of the multiplier HRS model (2) and its optimal solutions is problematic. We simply do not know what the constraints and optimal solutions of this model mean, except that it is the dual to the input-oriented envelopment model based on the HRS technology (1).

Let us show that the results obtained in this paper make the interpretation of the multiplier HRS model straightforward. As shown in Section 3.1, $\mathcal{T}_{\text {HRS }}$ is a polyhedral 
technology and its statement (1) is a special case of (4). Therefore, all results and interpretations obtained in this paper are applicable to this technology.

Let $\left(u^{*}, v^{*}, \sigma^{*}, \rho^{*}\right)$ be any optimal solution of program (2). Using formula (8) with $w^{*}=\left(\sigma^{*}, \rho^{*}\right)$ and noting the structure of the vector $U_{o}$ (see Section 3.1), we have $u_{0}^{*}=U_{o}^{\top} w^{*}=\rho^{*}$. We now have the following two alternative ways to interpret the optimal solution.

(i) By Theorems 3 and 4 , the triplet $\left(u^{*}, v^{*}, u_{0}^{*}\right)$ maximizes the efficiency ratio $\left(Y_{o}^{\top} u+u_{0}\right) / X_{o}^{\top} v$ of $\mathrm{DMU}_{o}$ with respect to the maximum of similar efficiency ratios of all DMUs in technology $\mathcal{T}_{\text {HRS }}$. This statement is true if the maximum of all such ratios is bounded above by 1 , as in the linear fractional model, or is unbounded, as in the maximin model.

(ii) By Theorem 1 and in line with the discussion in Sections 4.1 and 6.1, the vectors $v^{*}$ and $u^{*}$ are interpretable as the vectors of input and output prices that maximize the profit efficiency of $\mathrm{DMU}_{o}$ compared the entire HRS technology, subject to the cost-normalizing condition $X_{o}^{\top} v=1$. As shown in Section 4.1, for these most favorable prices, the maximum profit in the HRS technology is equal to $-u_{0}^{*}$. For the optimal value $\theta^{*}$ of the multiplier HRS model (2), the difference $1-\theta^{*}=-u_{0}^{*}-\left(Y_{o}^{\top} u^{*}-X_{o}^{\top} v^{*}\right)$ is equal to the shortfall of the profit made by $\mathrm{DMU}_{o}$ in comparison to the maximum profit $-u_{0}^{*}$. If $\theta^{*}=1$, there is no shortfall and $\mathrm{DMU}_{o}$ is profit efficient.

Both of the above interpretations clarify and substantiate the meaning of the optimal solution $\left(u^{*}, v^{*}, \sigma^{*}, \rho^{*}\right)$ as being the most favorable for $\mathrm{DMU}_{o}$ when it is benchmarked against all DMUs in technology $\mathcal{T}_{\text {HRS }}$. It is worth emphasizing that this interpretation involves all DMUs in the technology and becomes incorrect if we narrow it by benchmarking $\mathrm{DMU}_{o}$ against the observed DMUs only.

Furthermore, by Proposition 1, the equation

$Y^{\top} u^{*}-X^{\top} v^{*}+\rho^{*}=0$

defines a supporting hyperplane to technology $\mathcal{T}_{\text {HRS }}$ at the input radial projection $\left(\theta^{*} X_{o}, Y_{o}\right)$. As follows from Remark 4, if $\mathrm{DMU}_{o}$ is both input and output radial efficient (for which its strong efficiency is sufficient), the range of optimal values of $u_{0}^{*}=U_{o}^{\top} w^{*}=\rho^{*}$ defines the left-hand and right-hand scale elasticities $\varepsilon^{-}\left(X_{o}, Y_{o}\right)$ and $\varepsilon^{+}\left(X_{o}, Y_{o}\right)$ evaluated at $\mathrm{DMU}_{o}$ and its RTS characterization.

\subsection{A two-stage network technology}

Consider the following simple two-stage network DEA model (Färe and Grosskopf 2000). In the first stage of the production process, the vector of inputs $X \in \mathbb{R}_{+}^{m}$ is used to produce the vector of intermediate outputs $Z \in \mathbb{R}_{+}^{l}$. In the second stage, the same vector $Z$ is used as an input vector to produce the vector of final outputs $Y \in \mathbb{R}_{+}^{s}$.
Denote observed DMUs $\left(X_{j}, Z_{j}, Y_{j}\right)$, where $j=1, \ldots, n$. Let $\bar{X}, \bar{Z}$ and $\bar{Y}$ be the $m \times n, l \times n$ and $s \times n$ matrices whose columns are the vectors $X_{j}, Z_{j}$ and $Y_{j}, j=1, \ldots, n$, respectively.

Consider the VRS network technology $\mathcal{T}_{\mathrm{N}}$ stated in the input and final output dimensions $X$ and $Y$ only. It is defined as the set of all DMUs $(X, Y) \in \mathbb{R}_{+}^{m+s}$ for each of which there exist vectors $\lambda, \mu \in \mathbb{R}^{n}$, the vector of intermediate outputs $Z \in \mathbb{R}^{l}$ and slack vectors $S_{X} \in \mathbb{R}^{m}, S_{Y} \in \mathbb{R}^{s}$ and $S_{Z}^{1}, S_{Z}^{2} \in \mathbb{R}^{l}$ such that

$\bar{X} \lambda+S_{X}=X$,

$\bar{Y} \mu-S_{Y}=Y$,

$\bar{Z} \lambda-Z-S_{Z}^{1}=0$,

$\bar{Z} \mu-Z+S_{Z}^{2}=0$,

$1^{\top} \lambda=1$,

$1^{\top} \mu=1$,

$\lambda, \mu, Z, S_{X}, S_{Y}, S_{Z}^{1}, S_{Z}^{2} \geq 0$.

The above statement is a special case of the general statement of polyhedral technology (4) in which vector $\hat{\lambda}$ corresponds to the combined vector $\left(\lambda, \mu, Z, S_{Z}^{1}, S_{Z}^{2}\right)$ in (34). The equalities (34c)-(34f) are the parameter conditions (4c) in which $U_{o}=(0,0,1,1)^{\top}$, where the first two zero components are vectors of dimension $l$, and the last two components are scalars equal to 1 .

Consider assessing the input radial efficiency of $\mathrm{DMU}_{o}$ in technology $\mathcal{T}_{\mathrm{N}}$ by the envelopment program (5). Let vectors $v \in \mathbb{R}^{m}, u \in \mathbb{R}^{s}, \rho \in \mathbb{R}^{l}, \sigma \in \mathbb{R}^{l}$ and scalars $\tau$ and $\nu$ be dual to the constraints of this envelopment program. Note that the dual constraint to the primal vector $S_{Z}^{2}$ is the vector inequality $\sigma \leq 0$. Substituting $\sigma^{\prime}=-\sigma \geq 0$ and suppressing the "prime" symbol, we state the dual inputoriented multiplier program as follows:

$$
\begin{array}{ll}
\theta^{*}=\max & Y_{o}^{\top} u+\tau+\nu \\
\text { subject to } & X_{o}^{\top} v=1 \\
& \bar{Z}^{\top} \rho-\bar{X}^{\top} v+1 \tau \leq 0, \\
& \bar{Y}^{\top} u-\bar{Z}^{\top} \sigma+1 \nu \leq 0, \\
& -\rho+\sigma \leq 0, \\
& u, v, \sigma, \rho \geq 0, \tau, \nu \text { sign free. }
\end{array}
$$

Because of the complex structure of program (35), the direct interpretation of its optimal solutions appears problematic. This task is, however, easily resolved by the general results obtained in this paper. Let $\left(u^{*}, v^{*}, \sigma^{*}, \rho^{*}, \tau^{*}, \nu^{*}\right)$ 
be any optimal solution to program (35). By formula (8), we have $u_{0}^{*}=\tau^{*}+\nu^{*}$.

We can now repeat the two interpretations given for the HRS model in Section 11.1. Namely, (i) the triplet $\left(u^{*}, v^{*}, u_{0}^{*}\right)$ maximizes the efficiency ratio $\left(Y_{o}^{\top} u+u_{0}\right) / X_{o}^{\top} v$ of $\mathrm{DMU}_{o}$ against all DMUs in technology $\mathcal{T}_{\mathrm{N}}$; (ii) interpreting $v$ and $u$ as the vectors of input and output prices, the optimal prices $v^{*}$ and $u^{*}$ maximize the profit efficiency of this DMU compared to the entire technology $\mathcal{T}_{\mathrm{N}}$. The maximum profit across all DMUs in technology $\mathcal{T}_{\mathrm{N}}$ achieved for the prices $v^{*}$ and $u^{*}$ is equal to $-u_{0}^{*}=-\tau^{*}-\nu^{*}$.

In summary, as in any other polyhedral technology, the optimal solutions to the multiplier model (35) are the most favorable for the DMU under the assessment when it is benchmarked against the entire technology $\mathcal{T}_{\mathrm{N}}$.

Furthermore, by Proposition 1, the equation

$Y^{\top} u^{*}-X^{\top} v^{*}+\tau^{*}+\nu^{*}=0$

defines a supporting hyperplane to technology $\mathcal{T}_{\mathrm{N}}$ at the input radial projection $\left(\theta^{*} X_{o}, Y_{o}\right)$. As noted in Remark 4, if $\mathrm{DMU}_{o}$ is both input and output radial efficient, the range of the optimal values of the intercept $u_{0}^{*}=\tau^{*}+\nu^{*}$ defines the type of RTS exhibited by this DMU.

\subsection{Technologies with bounded measures}

The bounded CRS technology $\mathcal{T}_{\mathrm{B}}$ was introduced by Cooper et al. (2011) and further extended by Pastor et al. (2015). Let $X \in \mathbb{R}_{+}^{m}$ and $Y \in \mathbb{R}_{+}^{s}$ be the input and output vectors, respectively. Let $\left(X_{j}, Y_{j}\right), j=1, \ldots, n$, be the observed DMUs. Define $\bar{X}$ and $\bar{Y}$ the matrices of dimensions $m \times n$ and $s \times n$ whose columns are the vectors $X_{j}$ and $Y_{j}, j=1, \ldots, n$, respectively.

Technology $\mathcal{T}_{\mathrm{B}}$ assumes a bounded variant of CRS. It models the situation in which each input has a lower bound and each output has an upper bound. The vectors of lower input bounds and upper output bounds are denoted $\underline{x} \in \mathbb{R}_{+}^{m}$ and $\bar{y} \in \mathbb{R}_{+}^{s}$, respectively.

Technology $\mathcal{T}_{\mathrm{B}}$ is stated as the set of all DMUs $(X, Y) \in$ $\mathbb{R}_{+}^{m+s}$ for which there exist a vector $\lambda \in \mathbb{R}^{n}$ and slack vectors $S_{X}, S_{X}^{\prime} \in \mathbb{R}^{m}$ and $S_{Y}, S_{Y}^{\prime} \in \mathbb{R}^{s}$ such that

$$
\begin{aligned}
& \bar{X} \lambda+S_{X}=X, \\
& \bar{Y} \lambda-S_{Y}=Y, \\
& \bar{X} \lambda-S_{X}^{\prime}=\underline{x}, \\
& \bar{Y} \lambda+S_{Y}^{\prime}=\bar{y}, \\
& \lambda, S_{X}, S_{Y}, S_{X}^{\prime}, S_{Y}^{\prime} \geq 0 .
\end{aligned}
$$

It is clear that technology $\mathcal{T}_{\mathrm{B}}$ is a special case of the general polyhedral technology (4). Indeed, vector $\hat{\lambda}$ in (4) is the combined vector $\left(\lambda, S_{X}^{\prime}, S_{Y}^{\prime}\right)$ in (36). Conditions (36a) and (36b) correspond to conditions (4a) and (4b). The nonhomogeneous conditions (36c) and (36d) are parameter conditions (4c) in which vector $U_{o}$ is the combined vector $(\underline{x}, \bar{y})$.

Consider assessing the input radial efficiency of DMU $\left(X_{o}, Y_{o}\right) \in \mathcal{T}_{\mathrm{B}}$ by the multiplier program. Let vectors $v, u, \tilde{v}$ and $\tilde{u}$ be the dual vectors to the equalities (36a)-(36d), restated in the form used in the envelopment program (5), respectively. (Note that the dual constraint to the vector $S_{Y}^{\prime}$ is $\tilde{u} \leq 0$.) We have the following multiplier program:

$$
\begin{array}{ll}
\theta^{*}=\max & Y_{o}^{\top} u+\underline{x}^{\top} \tilde{v}+\bar{y}^{\top} \tilde{u} \\
\text { subject to } & X_{o}^{\top} v=1, \\
& \bar{Y}^{\top} u-\bar{X}^{\top} v+\bar{Y}^{\top} \tilde{u}+\bar{X}^{\top} \tilde{v} \leq 0, \\
& u, v, \tilde{v} \geq 0, \tilde{u} \leq 0 .
\end{array}
$$

Because of the complex structure of program (37), its meaning is not immediately clear and the interpretation of its optimal solutions appears problematic.

Let $\left(u^{*}, v^{*}, \tilde{u}^{*}, \tilde{v}^{*}\right)$ be any optimal solution to program (37). Using formula (8), define $u_{0}^{*}=\underline{x}^{\top} \tilde{v}^{*}+\bar{y}^{\top} \tilde{u}^{*}$. Based on the results obtained in this paper and similar to the interpretation of the HRS model given in Section 11.1, we interpret the optimal solution of program (37) as being the most favorable for $\mathrm{DMU}_{o}$ when it is benchmarked against the entire technology. More precisely, the optimal vectors of weights $u^{*}$ and $v^{*}$, together with $u_{0}^{*}$ defined above, maximize the efficiency ratio $\left(Y_{o}^{\top} u+u_{0}\right) / X_{o}^{\top} v$ of $\mathrm{DMU}_{o}$ against similar ratios of all DMUs in technology $\mathcal{T}_{\mathrm{B}}$. Alternatively, interpreting $u$ and $v$ (but not $\tilde{u}$ and $\tilde{v}$ ) as the vectors of prices, we conclude that the optimal vectors $u^{*}$ and $v^{*}$ maximize the profit efficiency of $\mathrm{DMU}_{o}$ when it is benchmarked against the entire technology $\mathcal{T}_{\mathrm{B}}$. The maximum profit for these optimal prices attained over the entire technology is equal to $-u_{0}^{*}=-\underline{x}^{\top} \tilde{v}^{*}-\bar{y}^{\top} \tilde{u}^{*}$.

Finally, by Proposition 1, we obtain the supporting hyperplane to technology $\mathcal{T}_{\mathrm{B}}$ at the input radial projection $\left(\theta^{*} X_{o}, Y_{o}\right)$ :

$$
Y^{\top} u^{*}-X^{\top} v^{*}+\underline{x}^{\top} \tilde{v}^{*}+\bar{y}^{\top} \tilde{u}^{*}=0 .
$$

As noted in Remark 4, if $\mathrm{DMU}_{o}$ is both input and output radial efficient, its RTS characterization is obtained by considering the full range of possible values of the optimal intercept $u_{0}^{*}=\underline{x}^{\top} \tilde{v}^{*}+\bar{y}^{\top} \tilde{u}^{*}$.

\section{References}

Afriat SN (1972) Efficiency estimation of production functions. Internat Econom Rev 13(3):568-598 
Allen R, Athanassopoulos A, Dyson RG, Thanassoulis E (1997) Weights restrictions and value judgements in data envelopment analysis: evolution, development and future directions. Ann Oper Res 73:13-34

Banker RD, Charnes A, Cooper WW (1984) Some models for estimating technical and scale inefficiencies in data envelopment analysis. Manage Sci 30(9):1078-1092

Banker RD, Thrall RM (1992) Estimation of returns to scale using data envelopment analysis. Eur J Oper Res 62(1):74-84

Briec W, Leleu H (2003) Dual representations of non-parametric technologies and measurement of technical efficiency. J Prod Anal 10(1):71-96

Chambers RG, Chung Y, Färe R (1998) Profit, directional distance functions, and Nerlovian efficiency. J Optim Theory Appl 98 (2):351-364

Charnes A, Cooper WW, Rhodes E (1978) Measuring the efficiency of decision making units. Eur J Oper Res 2(6):429-444

Cherchye L, De Rock B, Dierynck B, Roodhooft F, Sabbe J (2013) Opening the "black box" of efficiency measurement: Input allocation in multioutput settings. Oper Res 61(5):1148-1165

Cook WD, Hababou M, Tuenter HJH (2000) Multicomponent efficiency measurement and shared inputs in data envelopment analysis: an application to sales and service performance in bank branches. J Prod Anal 14(3):209-224

Cook WD, Tone K, Zhu J (2014) Data envelopment analysis: prior to choosing a model. Omega 44:1-4

Cooper WW, Pastor JT, Borras F, Aparicio J, Pastor D (2011) BAM: a bounded adjusted measure of efficiency for use with bounded additive models. J Prod Anal 35(2):85-94

Cooper WW, Thompson RG, Thrall RM (1996) Introduction: extensions and new developments in DEA. Ann Oper Res 66(1):3-45

Färe R, Grosskopf S (2000) Network DEA. Soc Econ Plan Sci 34 (1):35-49

Färe R, Grosskopf S (2004) New directions: efficiency and productivity. Kluwer Academic Publishers, Boston

Färe R, Grosskopf S, Logan J (1983) The relative efficiency of Illinois electric utilities. Resour Energy 5(4):349-367

Färe R, Grosskopf S, Lovell CAK (1985) The measurement of efficiency of production. Kluwer Academic Publishers, Boston

Färe R, Grosskopf S, Lovell CAK (1994). Production frontiers. Cambridge University Press: Cambridge

Färe R, Primont D (1995) Multi-output production and duality: theory and applications. Kluwer Academic Publishers, Boston

Førsund FR (2018) Economic interpretations of DEA. Soc Econ Plan Sci 61(10):9-15

Førsund FR, Hjalmarsson L (2004) Calculating scale elasticity in DEA models. J Oper Res Soc 55(10):1023-1038
Kuosmanen T, Post T, Sipilainen T (2004) Shadow price approach to total factor productivity measurement: with an application to Finnish grass-silage production. J Prod Anal 22(1-2):95-121

Mehdiloo M, Podinovski VV (2019) Selective strong and weak disposability in efficiency analysis. Eur J Oper Res 276 (3):1154-1169

Olesen OB, Petersen NC (1996) Indicators of ill-conditioned data sets and model misspecification in data envelopment analysis: an extended facet approach. Manage Sci 42(2):205-219

Olesen OB, Petersen NC (2015) Facet analysis in data envelopment analysis. In: Zhu J (ed) Data envelopment analysis: a handbook of models and methods. Springer Science + Business Media, New York, p. 145-190

Pastor JT, Aparicio J, Alcaraz J, Vidal F, Pastor D (2015) An enhanced BAM for unbounded or partially bounded CRS additive models. Omega 56:16-24

Podinovski VV (2001) DEA models for the explicit maximisation of relative efficiency. Eur J Oper Res 131(3):572-586

Podinovski VV (2004a) Bridging the gap between the constant and variable returns-to-scale models: selective proportionality in data envelopment analysis. J Oper Res Soc 55(3):265-276

Podinovski VV (2004b) Production trade-offs and weight restrictions in data envelopment analysis. J Oper Res Soc 55 (12):1311-1322

Podinovski VV (2016) Optimal weights in DEA models with weight restrictions. Eur J Oper Res 254(3):916-924

Podinovski VV, Athanassopoulos AD (1998) Assessing the relative efficiency of decision making units using DEA models with weight restrictions. J Oper Res Soc 49(5):500-508

Podinovski VV, Chambers RG, Atici KB, Deineko ID (2016) Marginal values and returns to scale for nonparametric production frontiers. Oper Res 64(1):236-250

Podinovski VV, Olesen OB, Sarrico SC (2018) Nonparametric production technologies with multiple component processes. Oper Res 66(1):282-300

Rockafellar RT (1970) Convex analysis. Princeton University Press, Princeton

Seiford LM, Thrall RM (1990) Recent developments in DEA: the mathematical programming approach to frontier analysis. $\mathbf{J}$ Econometrics 46(1-2):7-38

Shephard RW (1974) Indirect production functions. Mathematical Systems in Economics No. 10. Anton Hain, Meisenheim am Glan

Tone K (2001) A slacks-based measure of efficiency in data envelopment analysis. Eur J Oper Res 130(3):498-509

Zelenyuk V (2013) A scale elasticity measure for directional distance function and its dual: theory and DEA estimation. Eur J Oper Res 228(3):592-600 